\title{
Swift-XRT Follow-up of Gravitational-wave Triggers in the Second Advanced LIGO/Virgo Observing Run
}

\author{
Klingler, N. J.; Kennea, J. A.; Evans, P. A.; Tohuvavohu, A.; Cenko, S. B.; Barthelmy, S. D.; Beardmore, \\ A. P.; Troja, E.; Brown, P. J.; Burrows, D. N.
}

Total number of authors:

40

Published in:

Astrophysical Journal Supplement Series

Link to article, DOI:

10.3847/1538-4365/ab4ea2

Publication date:

2019

Document Version

Publisher's PDF, also known as Version of record

Link back to DTU Orbit

Citation (APA):

Klingler, N. J., Kennea, J. A., Evans, P. A., Tohuvavohu, A., Cenko, S. B., Barthelmy, S. D., Beardmore, A. P., Troja, E., Brown, P. J., Burrows, D. N., Campana, S., Cusumano, G., D'Ai, A., D'Avanzo, P., D'Elia, V., de Pasquale, M., Emery, S. W. K., Garcia, J., Giommi, P., ... Troja, E. (2019). Swift-XRT Follow-up of Gravitationalwave Triggers in the Second Advanced LIGO/Virgo Observing Run. Astrophysical Journal Supplement Series, 245(1), [15]. https://doi.org/10.3847/1538-4365/ab4ea2

\section{General rights}

Copyright and moral rights for the publications made accessible in the public portal are retained by the authors and/or other copyright owners and it is a condition of accessing publications that users recognise and abide by the legal requirements associated with these rights.

- Users may download and print one copy of any publication from the public portal for the purpose of private study or research.

- You may not further distribute the material or use it for any profit-making activity or commercial gain

- You may freely distribute the URL identifying the publication in the public portal 


\title{
Swift-XRT Follow-up of Gravitational-wave Triggers in the Second Advanced LIGO/ Virgo Observing Run
}

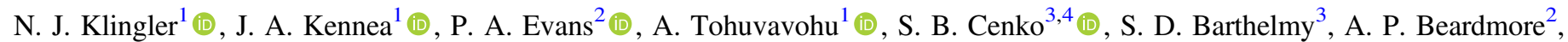

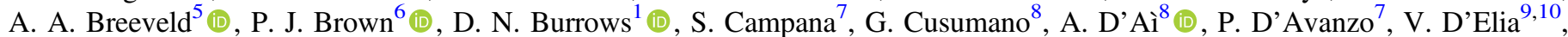
M. de Pasquale ${ }^{11}$, S. W. K. Emery ${ }^{5}$, J. Garcia ${ }^{12}$ (1) , P. Giommi ${ }^{10}$, C. Gronwall ${ }^{1,13}$, D. H. Hartmann ${ }^{14}$ (1) , H. A. Krimm ${ }^{15}$, N. P. M. Kuin ${ }^{5}$, A. Lien $^{16,17}$, D. B. Malesani ${ }^{18,19}$ (1) , F. E. Marshall ${ }^{3}$ (1) , A. Melandri ${ }^{7}$, J. A. Nousek ${ }^{20}$, S. R. Oates ${ }^{21}$, P. T. O’Brien ${ }^{2}$, J. P. Osborne ${ }^{2}$ (i) , K. L. Page (10, D. M. Palmer ${ }^{22}$ (1) , M. Perri ${ }^{9,10}$, J. L. Racusin ${ }^{3}$ (1) , M. H. Siegel ${ }^{20}$, T. Sakamoto ${ }^{23}$, B. Sbarufatti ${ }^{20}$, G. Tagliaferri ${ }^{7}$ (i) , and E. Troja ${ }^{3,24}$ (1)

${ }^{1}$ Department of Astronomy and Astrophysics, The Pennsylvania State University, 525 Davey Laboratory, University Park, PA 16802, USA; njk5441@psu.edu ${ }^{2}$ Department of Physics and Astronomy, University of Leicester, University Road, Leicester, LE1 7RH, UK

${ }^{3}$ Astrophysics Science Division, NASA Goddard Space Flight Center, Greenbelt, MD 20771, USA

${ }^{4}$ Joint Space-Science Institute, Computer and Space Sciences Building, University of Maryland, College Park, MD 20742, USA

${ }^{5}$ University College London, Mullard Space Science Laboratory, Holmbury St. Mary, Dorking, RH5 6NT, UK

${ }^{6}$ George P. and Cynthia Woods Mitchell Institute for Fundamental Physics \& Astronomy, Mitchell Physics Building, Texas A. \& M. University, Department of Physics and Astronomy, College Station, TX 77843, USA

${ }^{7}$ INAF-Osservatorio Astronomico di Brera, Via Bianchi 46, I-23807 Merate, Italy

${ }^{8}$ INAF-IASF Palermo, via Ugo La Malfa 153, I-90146, Palermo, Italy

9 INAF-Osservatorio Astronomico di Roma, via Frascati 33, I-00040 Monteporzio Catone, Italy

${ }^{10}$ Space Science Data Center (SSDC)—Agenzia Spaziale Italiana (ASI), I-00133 Roma, Italy

${ }^{11}$ Department of Astronomy and Space Sciences, Istanbul University, Beyzit 34119, Istanbul, Turkey

${ }^{12}$ Cahill Center for Astronomy and Astrophysics, California Institute of Technology, 1200 East California Boulevard, Pasadena, CA 91125, USA

${ }_{14}^{13}$ Institute for Gravitation and the Cosmos, The Pennsylvania State University, University Park, PA 16802, USA

${ }^{14}$ Department of Physics and Astronomy, Clemson University, Kinard Lab of Physics, Clemson, SC 29631, USA

${ }^{15}$ National Science Foundation, Alexandria, VA 22314, USA

${ }^{16}$ Center for Research and Exploration in Space Science and Technology (CRESST) and NASA Goddard Space Flight Center, Greenbelt MD, 20771 USA

${ }^{17}$ Department of Physics, University of Maryland, Baltimore County, 1000 Hilltop Circle, Baltimore, MD 21250, USA

${ }^{18}$ Dark Cosmology Centre, Niels Bohr Institute, University of Copenhagen, Juliane Maries Vej 30, DK-2100 Copenhagen Ø, Denmark

${ }^{19}$ DTU Space, National Space Institute, Technical University of Denmark, Elektrovej 327, DK-2800 Kongens Lyngby, Denmark

${ }^{20}$ Department of Astronomy and Astrophysics, The Pennsylvania State University, University Park, PA 16802, USA

${ }^{21}$ Department of Physics, University of Warwick, Coventry, CV4 7AL, UK

${ }^{23}$ Los Alamos National Laboratory, B244, Los Alamos, NM 87545, USA
Department of Physics and Mathematics, Aoyama Gakuin University, Sagamihara, Kanagawa, 252-5258, Japan

${ }^{24}$ Department of Physics and Astronomy, University of Maryland, College Park, MD 20742-4111, USA

Received 2019 August 20; revised 2019 October 10; accepted 2019 October 11; published 2019 November 15

\begin{abstract}
The Neil Gehrels Swift Observatory carried out prompt searches for gravitational-wave (GW) events detected by the LIGO/Virgo Collaboration (LVC) during the second observing run ("O2"). Swift performed extensive tiling of eight LVC triggers, two of which had very low false-alarm rates (GW170814 and the epochal GW170817), indicating a high confidence of being astrophysical in origin; the latter was the first GW event to have an electromagnetic counterpart detected. In this paper we describe the follow-up performed during $\mathrm{O} 2$ and the results of our searches. No GW electromagnetic counterparts were detected; this result is expected, as GW170817 remained the only astrophysical event containing at least one neutron star after LVC's later retraction of some events. A number of $\mathrm{X}$-ray sources were detected, with the majority of identified sources being active galactic nuclei. We discuss the detection rate of transient X-ray sources and their implications in the $\mathrm{O} 2$ tiling searches. Finally, we describe the lessons learned during $\mathrm{O} 2$ and how these are being used to improve the Swift follow-up of GW events. In particular, we simulate a population of gamma-ray burst afterglows to evaluate our source ranking system's ability to differentiate them from unrelated and uncataloged X-ray sources. We find that $\approx 60 \%-70 \%$ of afterglows whose jets are oriented toward Earth will be given high rank (i.e., "interesting" designation) by the completion of our second follow-up phase (assuming that their location in the sky was observed), but that this fraction can be increased to nearly $100 \%$ by performing a third follow-up observation of sources exhibiting fading behavior.
\end{abstract}

Unified Astronomy Thesaurus concepts: Gravitational waves (678); Astronomy data analysis (1858); Gamma-ray bursts (629); X-ray sources (1822); Surveys (1671); X-ray surveys (1824); Catalogs (205); Gravitational wave sources (677)

Supporting material: machine-readable table

\section{Introduction}

In 2017, the Advanced Laser Interferometer Gravitationalwave Observatory (aLIGO; LIGO Scientific Collaboration et al. 2015) and the Advanced Virgo detector (the Virgo
Scientific Collaboration; Acernese et al. 2015) collectively carried out the second observing run ("O2") in search of gravitational-wave (GW) events from 2016 November 30 to 2017 August 25. The GW triggers detected by the LIGO-Virgo 
Collaboration (LVC) were assigned parameters such as a falsealarm rate (FAR; characterizing the frequency at which noise with the same strength as the signal is expected to arise), whether the detected signal arose from a compact binary coalescence (CBC) or an unmodeled burst, ${ }^{25}$ and (for CBC triggers) the estimated distance of the merger and the masses of the initial compact objects. Triggers with an FAR of less than one per month were announced to electromagnetic (EM) follow-up partners who had signed a memorandum of understanding with the LVC. O2 resulted in the detection of GW170817, a binary neutron star (BNS) merger, which was the first GW event to have its EM counterpart (AT 2017gfo) detected (Abbott et al. 2017). The results of both $\mathrm{O} 1$ and $\mathrm{O} 2$ are summarized in the Gravitational-Wave Transient Catalog of Compact Binary Mergers (GWTC-1; Abbott et al. 2019b).

In addition to GWs, EM radiation is expected to be produced in both BNS and neutron star-black hole (NSBH) mergers, as was demonstrated in the case of GW170817 (at least for the BNS case). If Earth lies close to the axis of rotation of the compact objects (i.e., "on-axis"), the prompt emission from the resulting relativistic jet is expected to be visible as a short gamma-ray burst (sGRB; see, e.g., Berger 2014; D'Avanzo 2015; Beniamini et al. 2019). On longer timescales, the radioactive decay of heavy $r$-process nuclei can produce broadband EM radiation visible as a kilonova, regardless of the viewing angle (Eichler et al. 1989; Li \& Paczyński 1998; Metzger et al. 2010). Binary black hole (BBH) mergers are not typically expected to produce EM radiation (Kamble \& Kaplan 2013). However, it is theorized that under certain circumstances and with particular $\mathrm{BH}$ parameters (e.g., charged black $\mathrm{BHs}$, or if accreting or circumstellar material is present) $\mathrm{BBH}$ mergers may be able to produce EM radiation (see, e.g., Liu et al. 2016; Loeb 2016; Perna et al. 2016; Yamazaki et al. 2016; Zhang 2016), though this has yet to be observationally verified. Thus, to further our understanding of the physics of compact binary mergers, it is necessary to search for and study the EM counterparts to merger events following the detection of their GWs.

\subsection{The Neil Gehrels Swift Observatory}

The Neil Gehrels Swift Observatory (Gehrels et al. 2004) is a multiwavelength space-based NASA observatory whose primary mission is to detect and study GRBs and their afterglows in (soft) $\gamma$-rays, X-rays, ultraviolet, and optical wavelengths. The Burst Alert Telescope (BAT; Barthelmy et al. 2005) is designed to detect GRBs in the $15-350 \mathrm{keV}$ range using a coded aperture mask that covers a $\sim 2$ sr field of view (FOV). The X-ray Telescope (XRT; Burrows et al. 2005) is an imaging instrument operating in the $0.3-10 \mathrm{keV}$ range, with a circular 23!6-diameter FOV. The Ultraviolet/Optical Telescope (UVOT; Roming et al. 2005) covers the 1600-6240 $\mathrm{\text {band }}$ with six filters and the $1600-8000 \AA$ band with a white filter. Its FOV is square, with sides of $\sim 17^{\prime}$. Upon detecting a GRB, the BAT obtains its position (usually to within an accuracy of $1^{\prime}-4^{\prime}$ ), and the spacecraft autonomously slews to the GRB's position within minutes (if there are no observing constraints). The XRT and UVOT then observe the GRB afterglow and obtain arcsecond-scale localizations.

As mentioned by Evans et al. (2016b), in an ideal scenario the BAT would detect and localize the sGRB produced by a

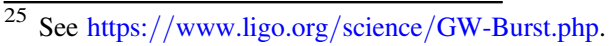

binary merger event independently of the detection of GW waves, promptly slew to the source, and detect the afterglow. However, GRBs are only seen if Earth lies within the jet's opening angle. ${ }^{26,27}$ The opening angles of GRB jets are not well measured. Their model-predicted values vary, with estimates currently placing the jet opening angle at $\theta_{j} \approx 3^{\circ}-10^{\circ}$, though some estimates are as high as $\theta_{j} \approx 20^{\circ}$ (see, e.g., Bloom et al. 2001; Frail et al. 2001; Berger 2014, and Berger et al. 2003). For a randomized distribution of sGRB jet axis orientations, the opening angles of $3^{\circ} / 10^{\circ} / 20^{\circ}$ correspond to $0.034 \% / 0.38 \% /$ $1.5 \%$ of sGRBs occurring on-axis (i.e., the fraction of the sky that would be encircled by both jet and counterjet for the above angles). Conversely, the gravitational waves emitted by merger events, though not isotropic, have a much weaker dependence on angle (the angular dependence is $\propto \cos (\theta / 2)$ ). Therefore, only a small fraction of detected BNS/NSBH merger events will produce sGRBs that are visible to Earth. Combined with the BAT's limited FOV (roughly $1 / 6$ of the sky at any time), simultaneous LVC-BAT detections are expected to be uncommon.

In the event of a GW trigger, Swift can cover substantial portions of the GW error region with the XRT and UVOT in relatively short amounts of time (see Evans et al. 2016b for a discussion of the Swift follow-up to O1 triggers). Since the allsky transient rate in X-rays (at Swift-XRT's sensitivity) is lower than that in the UV/optical bands (at UVOT's sensitivity; see, e.g., Kanner et al. 2013; Evans et al. 2016c), and with the XRT's FOV being larger than UVOT's, Swift's XRT also plays an important role in the search for and identification of EM counterparts to GW events. Since sGRB afterglows are not the only type of X-ray transients, as we search large areas of the sky it is important to consider the possibility of coincidental detections of unrelated X-ray transients and to quantify the rates of unrelated source detections in GW follow-up searches. The goal of this paper is to investigate and report on the rate of X-ray transients detected by Swift during O2, in particular, in the context of those detectable during the exposure times and timescales of the Swift GW follow-up procedures.

The paper is structured as follows. In Section 2 we present an overview of the general observing strategy used and information about the LVC GW triggers on which we followed up. In Section 3 we describe the data analysis techniques and source detection/flagging algorithms used. In Section 4 we present the results of the searches and properties of the population of detected sources. In Section 5 we discuss the implications of our results in the context of Swift follow-up of GW triggers in the Advanced LIGO/Virgo O3 run.

\section{Swift Response to GW events}

\subsection{Follow-up Observing Strategy}

The observing strategy employed in this campaign is the same as that which has been described in detail by Evans et al. (2016b, 2016c), so only a brief summary is provided here.

\footnotetext{
${ }^{26}$ The detection of prompt emission from sGRB 170817 A by the Fermi-GBM and INTEGRAL-SPI, despite being off-axis by $\sim 20^{\circ}$ (Ghirlanda et al. 2019), adds complications to this assumption by suggesting that at least some GRB jets may be structured and may release fainter X-ray emission over a wider angle. The prompt emission of sGRB 170817 was not seen by the BAT as occurred outside the BAT's FOV at the time.

${ }^{27}$ In addition to GRB jet emission, there may also be more isotropic X-ray emission (see, e.g., Sun et al. 2017).
} 
GW positional error regions can often encompass areas up to hundreds of $\mathrm{deg}^{2}$. Since the XRT FOV is only 23!6 in diameter, many pointings (tilings) are required to cover even a fraction of the higher-probability areas of the error region. In most cases covering the entire region within a reasonable time frame is not even feasible. CBCs are believed to occur in or near galaxies, (see, e.g., Fong et al. 2010; Tunnicliffe et al. 2014). Hence, a logical follow-up strategy is to convolve the LVC probability map and estimated distance of the triggers with the appropriate galaxy catalog (see Section 3.2 of Evans et al. 2016b and also Evans et al. 2019 for details). This method reduces the area that needs to be observed for each trigger by focusing on fields containing known galaxies that are possible hosts to the merger event. We used two catalogs: the Two Micron All Sky Survey (2MASS) Photometric Redshift Catalog (2MPZ; Bilicki et al. 2014) and the Gravitational Wave Galaxy Catalog (GWGC; White et al. 2011). For GW events where the mean estimated distance was $\leqslant 80 \mathrm{Mpc}$, we used the GWGC because it is more complete than 2MPZ in this regime. ${ }^{28}$ For the more distant events, $2 \mathrm{MPZ}$ was used since it is more complete. The selected galaxies (i.e., located within the LVC region and consistent with the estimated distance to the GW event) were then prioritized based on distance and luminosity (which is used as a proxy for mass), as the latter is expected to be an indicator of sGRB rate (see Fong et al. 2013). When using the GWGC, galaxies were weighted by their $B$-band luminosity, and when using 2MPZ, the $K$ band was used (i.e., the native bands of the catalogs; the impact of this is investigated in Figure 7 of Evans et al. 2016b). The (in) completeness of the catalogs is included in the convolution procedure (see Equations (5), (6), and (10) in the above paper). It is worth noting the caveat that there is a nonzero probability that the "correct" field will not be observed, due to the fact that we are not be able to cover anything near $100 \%$ of the GW probability region even after galaxy convolution. However, simulations by Evans et al. (2016c) show that the galaxy catalog convolution/targeted search method is more effective at detecting the EM counterpart before it fades than blindly searching the entire raw GW error region, which is far more time-consuming.

The overall observing strategy was to carry out three phases of observations. First (phase 1), a series of short (60 s) exposures were taken covering as much of the galaxy-mapconvolved GW error region as possible. This phase was designed with the intention of detecting an on-axis sGRB afterglow, if present, and continued for $\sim 2$ days. Next (phase 2), 2-3 days after the trigger, the GW error region was reobserved for $500 \mathrm{~s}$ per tile. These observations, which continued for up to 4 days, were optimized to search for the rising X-ray afterglow from an sGRB observed off-axis. Collectively, phases 1 and 2 are referred to as the "wide-area search" phase. Finally (phase 3), if no confirmed counterpart was found, any potentially interesting (i.e., unidentified) X-ray source was reobserved with exposures $>1 \mathrm{ks}$. Any such source found to be fading was reobserved repeatedly over the following days until it could be confirmed to be the counterpart, or ruled out as such.

In reality, this program was not always followed completely. The prolonged period of many short-exposure observations

\footnotetext{
${ }^{28}$ We also used this catalog for unmodeled burst events, which are only expected to be detectable within $100 \mathrm{Mpc}$ (LIGO Scientific Collaboration \& Virgo Scientific Collaboration 2019).
}

was unlike any previous use of Swift, and so the number of fields observed for the first few triggers was reduced to allow us to verify that this observing mode did not pose a risk to the spacecraft, as the large number of slews carried out in such a short time period was unprecedented. Even once this had been confirmed, we only carried out all three phases fully in one case, that of G275697 (see Table 1). For the BBH triggers, where an EM counterpart is not expected, we only carried out phase 1. Phase 2 was carried out for all BNS triggers except G275404 (because trigger G275697 occurred when phase 2 was due to start and we decided to prioritize the more nearby event) and G298048 (GW170817, for which the real counterpart was found and therefore phase 2 was unnecessary). For trigger G299232, only phases 1 and 2 were carried out. Additionally, we occasionally carried out targeted observations of potential counterparts reported in the GCN Circulars by other facilities.

\subsection{Follow-up Criteria}

GW trigger notices were issued by the LVC for any event with an FAR of $<1$ per month. These trigger notices also included an estimated $P_{\mathrm{NS}}$, the probability that the event involved at least one NS. We evaluated each trigger (convolved the sky map, estimated distance, and appropriate galaxy catalogs) and assigned each a "P400" value, which is the fraction of the probability region contained within the 400 most probable XRT fields taken from the galaxy-convolved sky map that were not Sun or Moon constrained by Swift. ${ }^{29}$ This essentially quantifies how much of the LVC probability region Swift-XRT can cover within 1 day.

The decision tree was set the following way: Swift would follow up an event only in the following cases:

1. For burst (unmodeled) triggers:

(a) if FAR $<1 / 6$ month $^{-1}$ and P400 $>0.2$.

2. For CBC triggers:

(a) if $P_{\mathrm{NS}}<0.25$ and $\mathrm{P} 400>0.5$;

(b) if $P_{\mathrm{NS}}>0.25$ (regardless of P400).

To test our follow-up response protocol, it was predetermined that the first two events in $\mathrm{O} 2$ would be followed up regardless of their qualifying criteria.

\section{Data Analysis}

The XRT data were automatically processed at the United Kingdom Swift Data Science Centre (UKSSDC) at the University of Leicester, using HEASOFT v6.22 and the latest CALDB available at the time of processing. Observation data were initially reprocessed using the XRTPIPELINE tool, which applied all necessary calibrations, filtering, and corrections. $^{30}$ Images and exposure maps of each observation were also created.

The basic steps of the XRT analysis of GW follow-up are as follows: (1) search for sources, (2) characterize sources, and (3) identify any potential counterparts to the GW trigger. Initial source detection was carried out during the wide-area search phase of the follow-up with the goal of finding sources of interest. The wide-area search phase includes the initial 60 and

\footnotetext{
29 A target is Sun/Moon constrained if it lies within $47^{\circ} / 23^{\circ}$ of the Sun/ Moon, respectively.

${ }^{30}$ For more details, see http://www.swift.ac.uk/analysis/ and https:// heasarc.gsfc.nasa.gov/ftools/caldb/help/xrtpipeline.html.
} 
Table 1

Overview of GW Triggers and Swift Follow-up in LVC O2

\begin{tabular}{|c|c|c|c|c|c|c|c|c|c|c|c|c|}
\hline Trigger ID & $\begin{array}{l}\text { Trigger Date } \\
(2017 ; \text { UT) }\end{array}$ & $\begin{array}{c}d_{\mathrm{est}} \\
(\mathrm{Mpc})\end{array}$ & $P_{\mathrm{NS}}$ & $\begin{array}{c}\text { Delay } \\
(\mathrm{ks})\end{array}$ & $\begin{array}{l}\text { Duration } \\
(\mathrm{ks})\end{array}$ & $\begin{array}{c}\text { Exposure } \\
(\mathrm{ks})\end{array}$ & $\begin{array}{l}\text { Num. } \\
\text { fields }\end{array}$ & $\begin{array}{c}\text { Area } \\
\left(\mathrm{deg}^{2}\right)\end{array}$ & $P_{\text {raw }}$ & $P_{\text {conv }}$ & $N_{\mathrm{XRT}}$ & $N_{\text {uncat }}$ \\
\hline G268556 & 01-04, 10:11:59 & $737 \pm 201$ & $0 \%$ & 50.3 & 1174 & 37 & 293 & 31.7 & $4.7 \%$ & $4.6 \%$ & 3 & 0 \\
\hline G270580 & $01-20,12: 31: 00$ & $\lesssim 100$ & (b) & 19.9 & 351 & 10 & 136 & 14.5 & $1.1 \%$ & $14 \%$ & 2 & 0 \\
\hline G274296 & $02-17,06: 05: 53$ & $\lesssim 100$ & (b) & & & & & & & & & \\
\hline G275404 & $02-25,18: 30: 21$ & $412 \pm 169$ & $100 \%$ & 17.3 & 1121 & 12 & 117 & 2.7 & $1.8 \%$ & $4.0 \%$ & 1 & 0 \\
\hline G275697 & $02-27,18: 57: 31$ & $193 \pm 61$ & $100 \%$ & 15.9 & 519 & 257 & 1408 & 171 & $16 \%$ & $31 \%$ & 58 & 16 \\
\hline G277583 & 03-13, 22:40:09 & $\lesssim 100$ & (b) & & & & & & & & & \\
\hline G284239 & $05-02,22: 26: 07$ & $\lesssim 100$ & (b) & & & & & & & & & \\
\hline G288732 & 06-08, 02:01:16 & $320 \pm 98$ & $0 \%$ & 62.8 & 29 & 9 & 4 & 0.5 & $0 \%$ & $0 \%$ & 0 & 0 \\
\hline G296853 & 08-09, 08:28:21 & $1086 \pm 302$ & $0 \%$ & & & & & & & & & \\
\hline G297595 & $08-14,10: 30: 43$ & $534 \pm 131$ & $0 \%$ & 31.2 & 517 & 113 & 643 & 68.0 & $24 \%$ & $36 \%$ & 41 & 15 \\
\hline G298048 & $08-17,12: 41: 04$ & $39 \pm 7$ & $100 \%$ & 3.3 & 9206 & 269 & 744 & 85.0 & $2.5 \%$ & $94 \%$ & 12 & 1 \\
\hline G298389 & $08-19,15: 50: 46$ & $\lesssim 100$ & (b) & & & & & & & & & \\
\hline G298936 & $08-23,13: 13: 58$ & $1738 \pm 477$ & $0 \%$ & & & & & & & & & \\
\hline G299232 & $08-25,13: 13: 37$ & $339 \pm 109$ & $100 \%$ & 11.1 & 748 & 156 & 653 & 75.9 & $8.3 \%$ & $16 \%$ & 40 & 19 \\
\hline
\end{tabular}

Notes. $d_{\text {est }}$ is the estimated distance to the GW event in Mpc. $P_{\text {NS }}$ is the probability that the event included at least one NS, for CBC triggers; "(b)" denotes that an event was a burst GW event, in which case (since little is known about their origin) the probability that they involve an NS cannot be determined. The delay is the interval between the GW trigger time and the time at which the first follow-up observation began. The duration is the time from the start of the first observation with Swift-XRT to the end of the last one. Swift-XRT was not observing the GW region for the entirety of this time, so the total exposure is given in the subsequent column. The area listed is corrected for the overlaps between adjacent tiles. $P_{\text {raw }}$ is the fraction of the LVC sky map that was enclosed by XRT observations, and $P_{\text {conv }}$ is the fraction of the galaxy-convolved sky map that was covered by XRT observations. $N_{\mathrm{XRT}}$ is the number of sources detected by the XRT in each follow-up search (only phases 1 and 2), and $N_{\text {uncat }}$ is the number (of $N_{\mathrm{XRT}}$ ) that are uncataloged.

${ }^{a}$ Trigger G275697 was retracted after the follow-up search was performed. Triggers G275404 and G299232 (although originally marked with $P_{\mathrm{NS}}=100 \%$ ) were later determined not to be real astronomical events (see Abbott et al. 2019a). For completeness, it is also worth noting that there were two additional GW triggers that were only detected in post-O2 analyses and consequently could not have been followed up within a reasonable amount of time: GW170729 and GW170818 (see Abbott et al. 2019b).

$500 \mathrm{~s}$ exposures of each field. The source detection procedure is an iterative process that involves sliding-cell source detection, background modeling, point-spread function (PSF) fitting, and a likelihood test to detect and localize the sources. This method was the same as that which has been used to produce the Swift X-Ray Point Source Catalog (1SXPS); it was described in detail by Evans et al. (2014). The pipeline assigned each detected source a quality flag, which characterizes the probability of the source being a spurious detection. Sources flagged as "good" have a $0.3 \%$ or less chance of being spurious (or false positive [FP]), "reasonable" sources have a 7\% FP rate, and "poor" sources have up to a 35\% FP rate. Considering both "good" and "reasonable" sources together yields a 1\% FP rate (as "good" sources are the most numerous), and all "good," "reasonable," and "poor" taken together result in an overall FP rate of roughly $10 \%$. The detected sources are manually verified for spurious detections that can arise from optical loading, stray light, extended emission, and/or thermal noise (which can result from the XRT detector getting too hot).

As previously noted by Metzger \& Berger (2012), when following up on LVC triggers, the major challenge is not only detecting an EM candidate counterpart but also discerning which, among the many sources detected, if any, is the actual EM counterpart to the GW event. Evans et al. (2015) discussed two methods of discerning an X-ray GRB afterglow from unrelated sources on the basis of brightness and/or fading behavior. A source can be a potential afterglow if it is bright enough that it should have been previously cataloged but has not been, in which case it has exhibited transient behavior. Additionally, sGRB X-ray afterglows fade on relatively short timescales, so a source may be a counterpart candidate even if it is below a catalog limit if it is fading rapidly. Therefore, the source characterization procedure is based on these considerations. The process accounted for the source brightness (in comparison to historical detections and flux limits), lightcurve behavior, and whether the source lies within $200 \mathrm{kpc}$ (in projection) of a known galaxy (see Bulik et al. 1999) with distance consistent (at the $3 \sigma$ level) with the distance estimate along that line of sight from the GW data. The flux limits were compared with those from the ROSAT All Sky Survey (RASS; Voges et al. 1999) and, where observations existed, from $X M M$-Newton observations (both pointed and slew surveys; Saxton et al. 2008) and the 1SXPS catalog (Evans et al. 2014).

Each source was placed into one of four rankings (as defined by Evans et al. 2016a), described below in decreasing order of importance.

Of highest priority ("rank 1") are afterglow candidates. Sources given this designation were either (1) uncataloged and at least $5 \sigma$ above the $3 \sigma$ upper limit from the RASS or 1SXPS or (2) a known X-ray source that is $5 \sigma$ above its cataloged flux. ${ }^{31}$ Afterglow candidates must also lie near (within $200 \mathrm{kpc}$ in projection of) a known galaxy (assuming that the source is at the distance of that galaxy).

Of subsequent importance are "interesting" sources ("rank 2 "). These are either (1) uncataloged and at least $3 \sigma$ above the $3 \sigma$ upper limit from the RASS/1SXPS or fading, or (2) known $\mathrm{X}$-ray sources at least $3 \sigma$ above their cataloged flux. Unlike afterglow candidates, an interesting source need not be near a known galaxy.

\footnotetext{
${ }_{31}$ The historical count rate/upper limits for both criteria were not derived from XRT data; they have been converted to equivalent XRT (PC mode) $0.3-10 \mathrm{keV}$ count rates using PIMMS (Portable Interactive Multi-Mission Simulator), assuming a typical active galactic nucleus (AGN) spectrum (absorbing hydrogen column density $N_{\mathrm{H}}=3 \times 10^{20} \mathrm{~cm}^{-2}$, and photon index $\Gamma=1.7$ ). The peak source fluxes were also obtained by converting from the peak count rates when assuming a typical AGN spectrum with the above-mentioned parameters.
} 
Next are uncataloged X-ray sources ("rank 3"). These were objects that were not previously cataloged in X-rays and that also meet none of the above criteria to differentiate them from field sources unrelated to a $\mathrm{GW}$ trigger.

Of least interest are known X-ray sources ("rank 4"). This category includes objects that have been detected in X-rays before and have a flux consistent with or below that from the previous observations. No further follow-up action is taken for these sources.

If a rank 1 source were detected at any point, the search would have been interrupted so the rank 1 source could be reobserved immediately. If the rank 1 source were determined not to be the afterglow, the search would be resumed. After the initial 60 and $500 \mathrm{~s}$ observations were completed, deeper follow-up observations were carried out ("phase 3"). Rank 2 sources were reobserved with deeper exposures of $\sim 5-6 \mathrm{ks}$. If no afterglow has been found at this point, rank 3 sources are then reobserved with $1 \mathrm{ks}$ observations. Some selected fields and/or sources were followed up for longer periods $(>10 \mathrm{ks})$ owing to target of opportunity (ToO) requests submitted by members of the astrophysical community. Phases 1-3 were only carried out in full for one trigger (G275697; see below).

\section{Results \\ 4.1. Follow-up Summary}

During O2, Swift carried out a follow-up search of seven CBC triggers (all of which had estimated distances $d_{\mathrm{est}}<1 \mathrm{Gpc}$ ) and one unmodeled burst. In total, 3998 XRT fields were observed, covering an area of $449.3 \mathrm{deg}^{2}$ (accounting for overlapping regions in XRT tilings; see, e.g., Figure 1 of Evans et al. 2015) in $863 \mathrm{ks}$ of observation time. A brief summary of the LVC O2 triggers and Swift follow-up searches is presented in Table 1.

It is worth noting that LVC trigger G275697 was subsequently retracted. Thus, the follow-up searches for this trigger are unique in that it is the only trigger for which we can be certain that we did not detect any counterpart (or that there was no counterpart to detect), since no actual astrophysical event took place.

LVC trigger G288732 did not meet our trigger criteria; however, a ToO request to observe a transient Fermi-LAT source spatially consistent with the GW error region was submitted, so Swift-XRT and UVOT began observing at 2017 June 08 at 19:27:20 (17.4 hr after the GW trigger). A four-point tiling was selected to cover the Fermi-LAT error region. The observations continued for $29 \mathrm{ks}$, until 2017 June 09 at 03:34:21, and gathered $9 \mathrm{ks}$ of observation data.

Swift also followed up LVC trigger G298048. This trigger was a BNS trigger, the famous GW170817. No early X-ray emission was detected; however, due to the detection of the optical transient AT 2017gfo (the first detection of an EM counterpart to a GW event), it was subsequently found that this GRB was off-axis and the afterglow rose later (see, e.g., Haggard et al. 2017; Margutti et al. 2017, 2018; Troja et al. 2017, 2018). This late-rising X-ray emission was not detected by the XRT, despite more than $50 \mathrm{ks}$ of exposure time; contamination from the X-ray-emitting host galaxy, combined with the relatively large PSF of XRT ( $9^{\prime \prime}$ half-energy width), made it impossible to obtain a solid detection of AT 2017gfo (i.e., distinguish the source's emission from that of its host galaxy).
More detailed descriptions of each trigger and follow-up searches are given in the Appendix.

\subsection{Detected Sources}

A total of 157 sources were detected in the wide-area search phase. Details for each source are listed in Table 2 (which can be found after the References section).

Four were flagged as "interesting" (rank 2). Among those, three were uncataloged X-ray sources, all of which exhibited significant fading $(4.1<\sigma<4.3)$, and only one of which was near $^{32}$ at least one galaxy (in this case, an AGN). Though the cataloged source did not exhibit any signs of fading, its peak flux had increased over its cataloged limit with a significance $>3 \sigma$.

A total of 51 sources were uncataloged (rank 3; i.e., they were not previously detected in X-rays). Of these, 35 lacked any nearby known galaxies within the range of distances compatible with their related GW triggers (though this designation does not mean that a source cannot be associated with a galaxy at a distance less than or greater than its respective $\mathrm{GW}$ trigger). Ten rank 3 sources were (or are positionally coincident with known) galaxies (or AGN candidates). Eight rank 3 sources exhibited evidence of fading: seven of these were of low significance $(1.1<\sigma<1.7)$, and only one was of high significance $(5.2 \sigma)$.

A total of 102 sources detected were previously cataloged X-ray (rank 4) sources. Of those, 41 were not located near any known galaxy that was consistent with the estimated distance of the GW trigger. Forty of the rank 4 sources were identified as (or are positionally coincident with known) galaxies (or AGN candidates). Eight sources exhibited slight evidence of fading $(1.1<\sigma<1.4)$. Of the fading sources, four are AGNs (or AGN candidates), one is a star, one is an eclipsing binary, and the remaining two are unknown.

A total of 153 of the sources were of the "good" detection quality flag, and four were of the "reasonable" quality flag. Of the latter, three were rank 3 (and unidentified) sources, and one was a rank 4 (known X-ray) source.

\section{Discussion}

\subsection{What We Found}

In Figure 1 we present a histogram of the peak fluxes of the detected sources (left panel) and a $\log N-\log S$ plot (right panel). In Figure 2 we present a histogram of the peak fluxes, but separated by source rank (ranks 3 and 4 are shown; no rank 1 sources and only four rank 2 sources were detected). In Figure 3 we plot the peak fluxes of all sources versus the theoretical exposure time required to detect each source. From these, we can see that few sources with peak fluxes $\lesssim 6 \times$ $10^{-13} \mathrm{erg} \mathrm{cm}^{-2} \mathrm{~s}^{-1}$ were detected.

There are two sources that should have been detected in the initial $60 \mathrm{~s}$ exposures with $\approx 100 \%$ confidence but were not (those that are enclosed within the dashed pink region). If $90 \%$ confidence is considered (i.e., those slightly below the horizontal pink line), then there are a couple more sources of

\footnotetext{
32 The galaxies are checked using the GWGC and $2 \mathrm{MPZ}$ catalog. To be considered "nearby" a galaxy, a source must be within $200 \mathrm{kpc}$ of a galaxy (in projection, at that distance), assuming the source is at the distance of the galaxy. Thus, a source can be marked as "having 0 nearby galaxies" but be coincident with a galaxy that is outside the range of estimated distances of the GW trigger, in which case it would be unrelated to the trigger.
} 

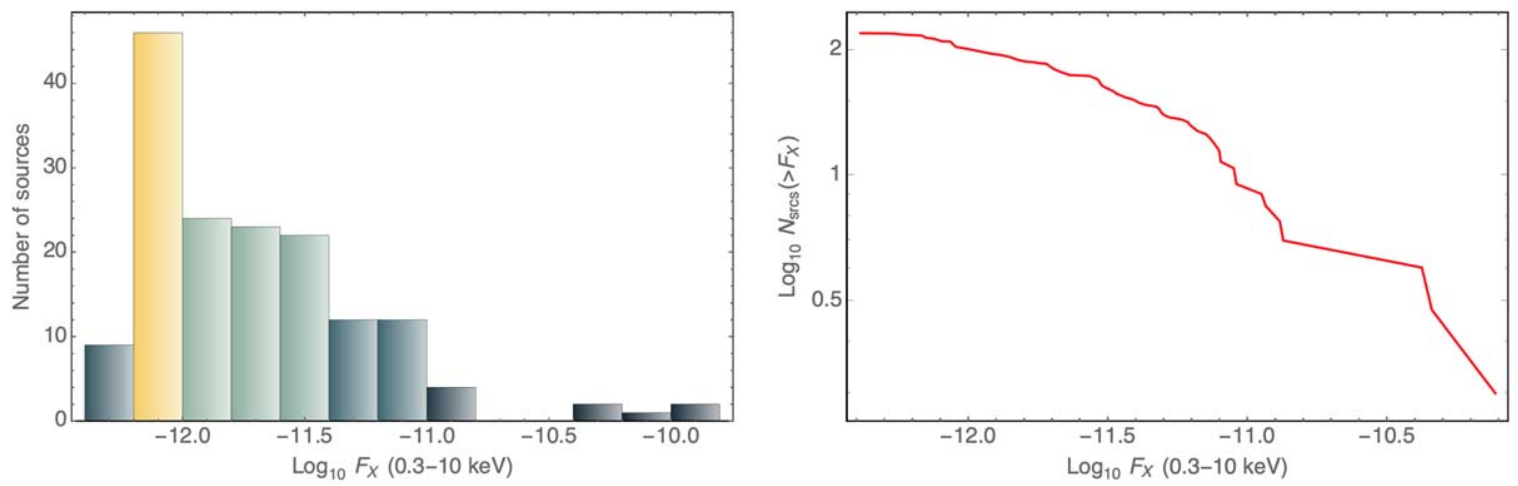

Figure 1. Left: histogram of the X-ray fluxes $F_{\mathrm{X}}$ of sources detected in the wide-area search phase (phases 1 and 2). Right: $\log N$ - $\log S$ plot of the population of detected sources in the wide-area search phase. (Here we represent X-ray flux with $F_{X}$ instead of the traditional $S$ ). Fluxes are in units of erg $\mathrm{cm}^{-2} \mathrm{~s}^{-1}$.

this type. This indicates that these sources have risen in brightness considerably between the initial exposure (phase 1; $60 \mathrm{~s}$ ) and a second deeper exposure (phase 2; $500 \mathrm{~s}$ ) in timescales of $<5$ days. These two prominent sources (i.e., within the pink region), 1SXPS J133553.6-341744 (trigger G298048, rank 4) and 3XMM J023819.7-521132 (trigger G297595, rank 4), are close to ( $<5^{\prime \prime}$ from) ESO $383-35$ and ESO 198-24, respectively, which are both type 1 Seyfert galaxies (AGNs).

This has two implications. The first is that a "late" detection of a bright source can be an indicator of variability and could be used to identify potential GW counterparts (i.e., sGRBs whose jets were initially not on-axis but that later widened). The second is that the spacing of our 60 and $500 \mathrm{~s}$ follow-up observations is consistent with the timescales of AGN variability, and we are able to detect such behavior. Indeed, in X-rays, AGNs are known to be able to vary down to timescales on the order of $1 \mathrm{hr}$ (see, e.g., Middei et al. 2017). Since typically the area searched lies outside the Galactic plane (as sGRBs/GW events have an isotropic distribution in the sky), it is reasonable to assume that the majority of X-ray sources seen will be AGNs. More than half $(7 / 10)$ of the cataloged X-ray sources detected in our follow-up exhibiting variability are identified as AGNs. The other three of the remaining cataloged and identified sources are stars (one of which is XMMSL1 J114247.5-354904, the counterpart to $\mathrm{V}^{*}$ V752 Cen/HD 101799: an eclipsing binary with an $8 \mathrm{hr}$ period; Sisteró \& Castore de Sisteró 1974). Thus, a point of consideration in future GW follow-up campaigns is that if a GW counterpart occurred in an X-ray-active galaxy, the automated source ranking system would classify it as a known source getting brighter. Since AGNs commonly vary on timescales comparable to our follow-ups, differentiating between an AGN "hiccup" and a transient occurring in a galaxy with an active nucleus will be challenging.

About $13 \% \pm 3 \%$ of the sources detected in the wide-area search phase $(21 / 157)$ exhibited fading behavior with a significance $\geqslant 1 \sigma$. It is worth noting that, assuming Gaussian errors, we would expect $16 \%$ of constant sources to be identified as fading at a level $\geqslant 1 \sigma$; therefore, the observed fraction of fading sources is consistent with what is expected from a population of constant sources. Two uncataloged sources with fluxes slightly above the RASS limit were detected. The flux and uncertainty of the first source (trigger G275697, rank 3), Swift J213954.9+444551.1, places it at $0.9 \sigma$ above the RASS limit; however, it exhibited fading behavior with $1.7 \sigma$. The second source (trigger G298048, rank
3), Swift J132507.3-323814.4, at $1.9 \sigma$ above the RASS limit, is coincident with 2MASX J13250705-3238129 and the radio source VBM97 J1325-3238, which are coincident with the galaxy cluster A3556, and thus this source is likely an AGN. Considering the two most significant rising sources (those above the pink line in Figure 3), the two above the RASS limit, and the cataloged interesting source, the fraction of sources exhibiting transient behavior in the wide-area search phase is $17 \% \pm 3 \%(26 / 157)$.

Although Swift did not find any counterparts, except for trigger G298048 (GW170817), Swift was not expected to find any, as none of the other triggers in $\mathrm{O} 2$ contained a BNS merger, triggers G275404 and G299232 were subsequently determined not to be real (Abbott et al. 2019a), and (for most triggers) only a small fraction of the probability regions were covered.

\subsection{Looking Forward: What We Can Expect}

Using the peak fluxes of all 157 sources detected and the XRT sensitivity curves (Figure 3), we can calculate the theoretical exposure times needed to detect each source at $50 \%$ and $90 \%$ confidence, ${ }^{33}$ and from that we can construct a cumulative distribution function (CDF) for the probability of detecting a source as a function of exposure. ${ }^{34}$ We divide the CDF by the total area covered in the GW searches $\left(449.3 \mathrm{deg}^{2}\right)$ and scale it to the area covered by the XRT FOV $\left(0.1215 \mathrm{deg}^{2}\right)$. In Figure 4 we plot this CDF, which shows the probability of detecting at least one source (per unit area corresponding to the XRT FOV) as a function of exposure time. After a $560 \mathrm{~s}$ exposure, the probabilities of detecting at least one source at $50 \%$ and $90 \%$ confidences in an XRT FOV are approximately 0.035 and 0.018 , respectively. Therefore, given the low rate of serendipitous X-ray sources expected, the probability of the GW counterpart being blended with an unrelated source is low (assuming that the counterpart is not in an X-ray-active host).

\subsubsection{Source Detection Rates}

Trigger G275697 was notable in that it was the first trigger for which phase 1 was carried out in full (after which, it is worth noting, no negative effects on the spacecraft from the

\footnotetext{
${ }^{33}$ In other words, the exposure times corresponding to a $50 \%$ and $90 \%$ chance of detecting a source (assuming that the source's count rate can be described by a Poissonian process).

${ }^{34}$ It is worth noting that the sensitivity at short exposures may not extrapolate perfectly.
} 

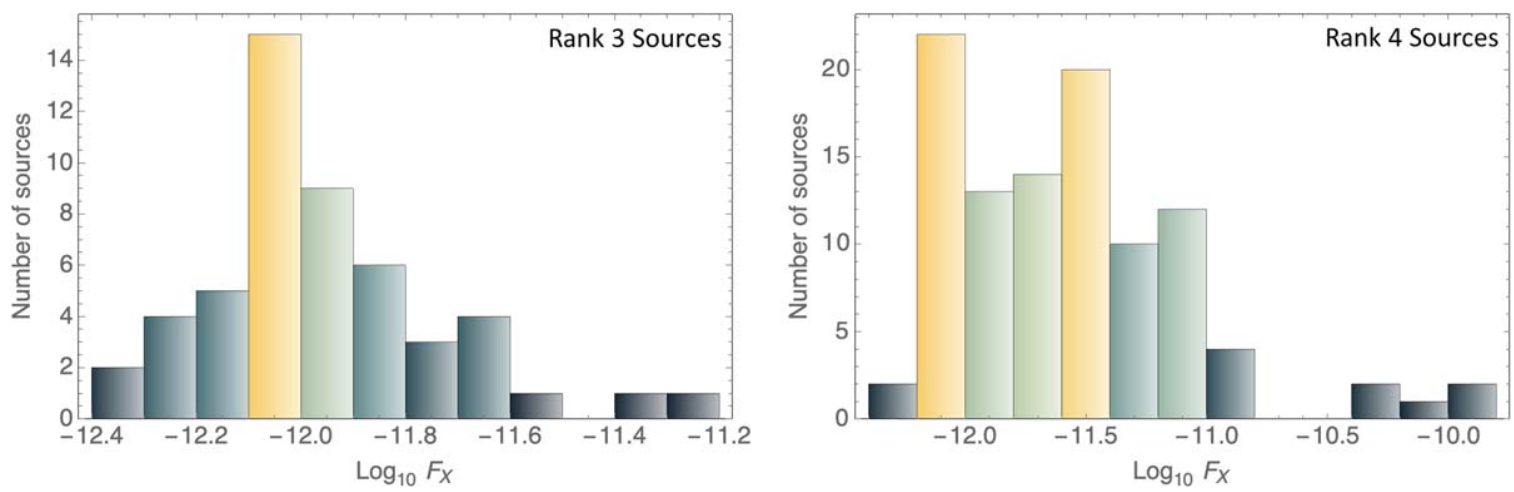

Figure 2. Histograms of the X-ray $(0.3-10 \mathrm{keV})$ fluxes $F_{X}$ of sources detected in the wide-search phase (phases 1 and 2$)$ separated by source rank. Left: rank 3 ; right: rank 4. Fluxes are in units of erg $\mathrm{cm}^{-2} \mathrm{~s}^{-1}$.

large number of slews were observed). It was also the only trigger for which phases 1,2 , and 3 were carried out in full. Lastly and of most importance, as this trigger was retracted, it is the only follow-up search for which we can be certain that there was no actual afterglow. Using this population of definitively unrelated sources, we can place (crude) limits on the detection rates of serendipitous sources for each rank. We can expect rank 1 and 2 sources to be detected at a rate of $<0.0175 \pm 0.0101 \mathrm{per} \mathrm{deg}^{2}$, rank 3 sources at a rate of $0.0936 \pm 0.0234$ per $\mathrm{deg}^{2}$, and rank 4 sources at a rate of $0.2280 \pm 0.0365$ per $\mathrm{deg}^{2}$. If we also consider triggers G297595 (LVC 170814) and G299232 (LVC 170825), the other two triggers for which phase 2 observations were carried out, we can refine our estimates as follows: ${ }^{35}$ we can expect to detect rank 1 and 2 sources at a rate of $<0.0127 \pm 0.0064$ per $\mathrm{deg}^{2}$, rank 3 sources at a rate of $0.1588 \pm 0.0225 \mathrm{per} \mathrm{deg}^{2}$, and rank 4 sources at a rate of $0.2699 \pm 0.0293$ per $\operatorname{deg}^{2}$ (assuming that none of the sources from triggers G297595 or G299232 were an actual counterpart).

\subsection{Evaluation of Source Ranking Criteria}

To investigate our efficacy in distinguishing an afterglow from unrelated X-ray sources (i.e., our ability to assign afterglows rank 1 or 2), we performed a simulation of afterglows and ran those with fluxes above our detection limits through our source ranking algorithm. We used afterglowpy ${ }^{36}$ (Ryan et al. 2019), a Python module that produces GRB light curves using the models of van Eerten et al. (2010) and van Eerten (2018), to simulate 10,000 sGRB events.

For all simulations, we used the typically assumed electron thermal energy fraction $\epsilon_{e}=0.1$ (which is consistent with recent observational results; see, e.g., Beniamini \& van der Horst 2017) and $d=120 \mathrm{Mpc}$ (the expected average distance of BNS mergers in O3). For the other parameters, we used the values obtained from X-ray observations of sGRBs (and those derived from observationally determined parameters) from Fong et al. (2013). We randomly selected electron spectral energy distribution slope $p$ and jet energy $E_{\text {jet }}$ from the list of observed values (Table 3 of Fong et al. 2015). For the circumburst number density $n$ we randomly selected a value in the range of $(0.6-15) \times 10^{-3} \mathrm{~cm}^{-3}$, which corresponds to the

\footnotetext{
$\overline{35}$ We do not consider triggers for which only phase 1 (60 s) observations were carried out, as Swift-XRT cannot reach fluxes below the RASS limit in such short exposures, and therefore we do not expect to detect uncataloged sources.

36 https://github.com/geoffryan/afterglowpy
}

best-fit value for GW/GRB 170817 (a rather low value) and the upper range of values considered typical, and for $\epsilon_{B}$ we selected a random value between 0.01 and 0.1 (thus sampling the range of typical values). For the off-axis (viewing) angle $\theta_{\text {obs }}$, we sampled from the expected distribution of off-angles detectable by LVC (see Equation (28) of Schutz 2011). For each simulated event, we calculated the $0.3-10 \mathrm{keV}$ flux at two times corresponding to the typical elapsed times between the trigger, $t_{0}$, and our phase 1 and phase 2 observations, $t_{1}, t_{2}$. For $t_{1}$ we picked a random time between $t_{0}+1.5 \mathrm{hr}(1.1 \mathrm{hr}$ for the average time between ground station passes during which we can upload tiling plans, and $0.4 \mathrm{hr}$ for roughly half of a Swift orbit) and $t_{0}+1.5$ days $+1.5 \mathrm{hr}$ (i.e., within the first half of phase 1; we assume that the afterglow will lie in the higher-probability areas, which we observe first). For $t_{2}$ we picked a random time between $t_{0}+3$ days $+1.5 \mathrm{hr}$ (the start of phase 2) and $t_{0}+4$ days $+1.5 \mathrm{hr}$ (the second day of phase 2 ). If the afterglow's flux at $t_{1}$ and/or $t_{2}$ reaches our detection thresholds for $80 \mathrm{~s}$ and/or $500 \mathrm{~s}$ tilings (the exposure times we will use in $\mathrm{O} 3$ follow-up; thresholds are $4.5 \times 10^{-12} \mathrm{erg} \mathrm{s}^{-1}$ $\mathrm{cm}^{-2}$ and $8 \times 10^{-13} \mathrm{erg} \mathrm{s}^{-1} \mathrm{~cm}^{-2}$, respectively), we simulated the observed XRT count rate in PIMMS using that sGRB's flux, power-law slope $p$, and an $N_{\mathrm{H}}$ randomly selected between $10^{21}$ and $10^{22} \mathrm{~cm}^{-2}$ (the approximate range of typical values; see Figure 7(a) of Evans et al. 2009 ${ }^{37}$ ). Our source ranking procedure (described above) takes into account the RASS upper limits, which can vary by R.A./decl., so we obtained a "typical" RASS upper limit by taking the average of limits at three high-probability regions of each LVC trigger we performed tilings for. We gathered these limits from the ESA Upper Limits Server, ${ }^{38}$ and we converted them into a 0.3-10 keV XRT count rate (we found the average value to be 0.023 counts s${ }^{-1}$ ). We tested the above simulations with the option for jet spreading turned off (with initial Lorentz factor $\gamma_{0}=1000$, the recommended value) and turned on (with initial Lorentz factor $\gamma_{0}$ set to infinity, the default value), and our results were the same (suggesting that jet spreading does not have a noticeable effect on the observed fluxes over the elapsed times we used for $t_{1}$ and $t_{2}$ ). For the jet, we assumed a Gaussian structure with a wing truncation angle of $20^{\circ} .1$ (the default value) and reperformed the entire simulation using each of the following opening angles $\theta_{j}: 5^{\circ}, 10^{\circ}$, and $20^{\circ}$ (since this not-

\footnotetext{
37 See https://www.swift.ac.uk/xrt_live_cat/\#figureDiv.

38 http://xmmuls.esac.esa.int/upperlimitserver/
} 


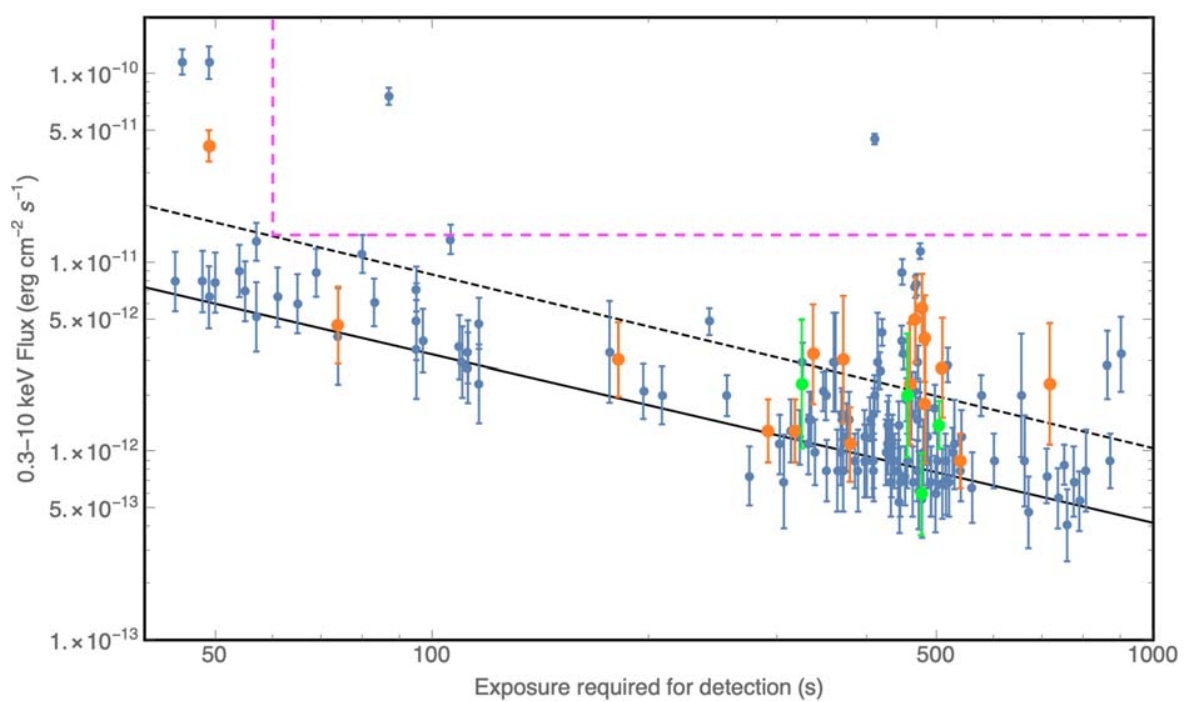

Figure 3. Plot of the peak fluxes of all sources vs. the theoretical exposure time required to detect each source. The black solid/dashed lines represent the XRT sensitivity as a function of exposure time, at the 50\%/90\% confidence levels (from 1SXPS), respectively (note that the sensitivity "lines" are rather curves, since they are shown in log space). The plotted points represent the peak fluxes of each source (with their corresponding $1 \sigma$ uncertainties; $y$-axis) vs. the effective exposure time needed to detect a source at its flux. Sources that exhibited slight fading $(1<\sigma \leqslant 2)$ are highlighted in orange, and sources that exhibited more significant fading (at least $>2 \sigma$ ) are highlighted in green (though these sources all have $>4 \sigma$ significance). Some sources have exposures for detection $>560 \mathrm{~s}$, which were found in the overlapping regions of adjacent tilings. The two sources fully enclosed within the dashed pink dashed lines are sources not detected in the initial $60 \mathrm{~s}$ exposures, but they should have been had they been this bright (i.e., at their peak flux) when those observations were carried out.

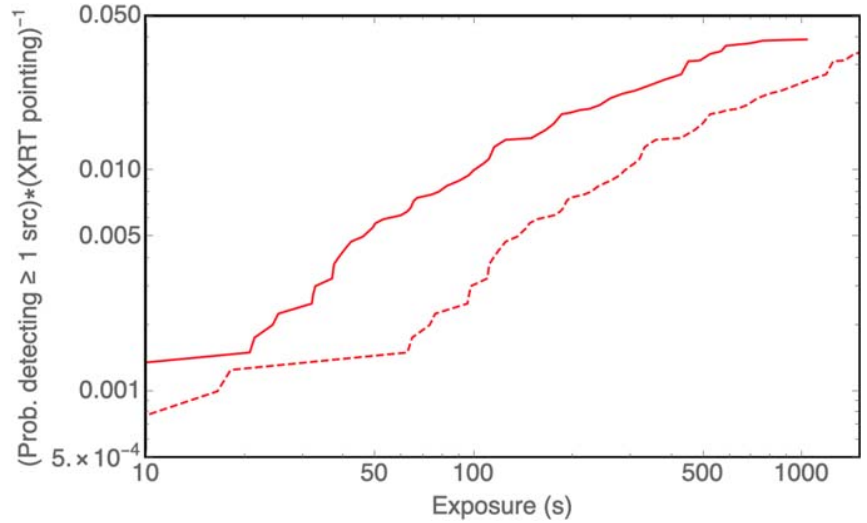

Figure 4. Plot of the probability of detecting at least one source per XRT field of view as a function of exposure time. The solid curve represents detections at $50 \%$ confidence, and the dashed curve represents detections at $90 \%$ confidence.

well-known parameter has the greatest effect in determining the detectability of afterglows).

For $\theta_{j}=5^{\circ}$ (in which case $0.095 \%$ of GRBs will occur onaxis), we found that 226 afterglows (2.3\%) would be detectable (assuming that the field they are located in is observed) and that $43 \%$ of the detectable afterglows would never exceed rank 3 (i.e., they were classified as rank 3 in both phases 1 and 2). Among the 226, 120 afterglows were detectable in both phases, 47 afterglows in only phase 1 , and 59 afterglows in only phase 2. For $\theta_{j}=10^{\circ}$ (in which case $0.38 \%$ of GRBs will occur onaxis), we found that 583 afterglows (5.8\%) would be detectable and that $40 \%$ would never exceed rank 3 . Among the 583, 398 afterglows were detectable in both phases, 77 afterglows in only phase 1 , and 108 in only phase 2 . For $\theta_{j}=20^{\circ}$ (in which case $1.5 \%$ of GRBs will occur on-axis), we found that 1399 afterglows (14\%) would be detectable and that $30 \%$ would never exceed rank 3. Among the 1399, 1030 afterglows were detectable in both phases, 201 in only phase 1, and 168 in only phase 2. We also found that very few of the detectable afterglows will exhibit fading with a significance below $1 \sigma$; for $\theta_{j}=2.5,5^{\circ}$, and $10^{\circ}$, the rates were $4.2 \%, 1.1 \%$, and $0.3 \%$, respectively. Although $16 \%$ of constant sources should be expected to exhibit fading with a significance $\sigma \geqslant 1$ purely by chance (which is in agreement with the observed number of "fading" rank 3 sources: $8 / 51=16 \%$ ), real afterglows will almost always exhibit fading at this significance or higher across timescales corresponding to the spacing of our phase 1 and phase 2 observations. We will use this finding to prioritize phase 3 follow-up of sources with these characteristics.

\subsection{Changes for $\mathrm{O3}$}

The lessons from $\mathrm{O} 2$ do not necessitate any major changes from the XRT perspective. To date, we have focused Swift follow-up on the XRT, but GW170817 has shown that UVOT is a crucial discovery instrument (see Evans et al. 2017). However, a challenge can arise from the UVOT FOV being smaller than that of the XRT. To address this, for CBC triggers in O3, we have modified our target selection criteria. Now, fields are initially selected based on tiling the XRT (as it is not practical to tile the smaller UVOT), but any field that was selected because it contained a potential host galaxy can now be offset or split into multiple fields. This will ensure that the galaxy or galaxies in question fall entirely within the UVOT FOV. We have also made changes to the way in which selected fields are organized into an observing plan in order to achieve greater efficiency (in that less time is spent slewing) in the coverage of the LVC region.

As another minor change, the exposure time of phase 1 observations has been increased from 60 to $80 \mathrm{~s}$. Postprocessing of the initial $60 \mathrm{~s}$ observations occasionally reduces their effective exposes to less than $60 \mathrm{~s}$ (e.g., the removal of periods of high background), and/or exposures can also be 
shortened owing to uncertainties in the estimated durations of slews. The extra allocated $20 \mathrm{~s}$ will guarantee that all initial exposures reach effective times of at least $60 \mathrm{~s}$.

In regard to the evaluation of our source ranking criteria, although we will not be changing source ranking criteria, we will begin to include the significance of any fading behavior in detected sources in GCN notices. This will allow us to differentiate "interesting" rank 3 sources from the "uninteresting" ones, without lowering the threshold criteria for rank 2 sources. We would like to remind the astronomical community that real afterglows (at typical distances) have an estimated $30 \%-40 \%$ of being assigned rank 3 , and that in almost all cases we should be able to detect fading at at least $1 \sigma$ significance. Therefore, fading rank 3 sources should not be neglected in potential multiwavelength follow-up, especially in the absence of higher-rank targets.

Lastly, in future searches we will be able to make use of an additional tool to detect an afterglow: the Swift Gravitational Wave Galaxy Survey (SGWGS). For this campaign, we selected the 20,000 most luminous galaxies in the GWGC (i.e., within $100 \mathrm{Mpc}$ ) and are seeking to observe each for at least $1 \mathrm{ks}$. The 20,000 galaxies chosen equate to roughly $50 \%$ of the total luminosity in the GWGC. This gives us beneficial pre-imaging information that will allow us to determine the number of preexisting sources (which will make the identification of transients more reliable in those fields) and also will give us a set of reference images for calculating upper limits or performing UVOT difference imaging. This will also drastically reduce the number of uncataloged X-ray and UV/optical sources that a potential afterglow might be miscategorized as. Currently, about $50 \%$ of the selected galaxies have been imaged. The details of the SGWGS will be discussed in a later publication (A. Tohuvavohu et al. 2019, in preparation).

\section{Summary}

We reported on the follow-up searches carried out by the Neil Gehrels Swift Observatory for GW triggers in the second LVC observing run (O2) in 2017 and described the search strategy and source analysis methods employed. The SwiftXRT observed 3998 fields containing potential host galaxies to the GW events, covering a total of $449 \mathrm{deg}^{2}$. We described the properties of the 157 (noncounterpart) X-ray sources detected and the detection rates of sources of each rank. We found that $17 \%$ of the sources exhibited variability, with the majority (7/ 10) of identified variable sources being AGNs. Thus, we expect the rate of unrelated transients to be manageably low. We reexamined the Swift follow-up strategy and our source ranking criteria and discuss minor improvements to be implemented.

N.J.K. would like to acknowledge support from NASA grant 80NSSC19K0408. P.A.E., A.P.B., J.P.O., and K.L.P. acknowledge support from the UK Space Agency. S.R.O. gratefully acknowledges the support of the Leverhulme Trust Early Career Fellowship. The Dark Cosmology Centre was funded by the Danish National Research Foundation. D.B.M. is supported by research grant 19054 from Villum Fonden. A.D. acknowledges financial contribution from the agreement ASI-INAF n.2017-14-H.0. The authors would also like to thank the anonymous referee for their useful suggestions, which helped to improve the paper.

Facility: the Neil Gehrels Swift Observatory.
Software: HEAsoft (v6.22; HEASARC 2014), afterglowpy (v0.6.4; Ryan et al. 2019).

\section{Appendix \\ Details of LVC Triggers and Swift Follow-up in $\mathrm{O2}$}

In this appendix, we list provide descriptions of each LVC trigger and information regarding Swift's response, as well as a list of all X-ray sources detected in our follow-up campaign (see Table 2).

\section{A.1. G268556/LVC 170104}

A CBC trigger occurred at 10:11:59 UT, with an estimated distance $d=737 \pm 201 \mathrm{Mpc}$. The first pre-planned science target (PPST) list was uploaded at 21:37 UT, and the first Swift observation occurred at T0+50.32 ks. Of the 600 planned tiles, 293 were observed, covering $4.7 \%$ of the raw (and $4.6 \%$ of the convolved) LVC probability region. A total of $30 \mathrm{X}$-ray sources were detected: 6 known (rank 4) sources and 24 unlikely afterglows (rank 3 sources).

ATLAS identified a possible counterpart (ATLAS17aeu) that Swift followed up and found to be fading in X-rays (GCNs 20390, 20415). UVOT found nothing above $3 \sigma$ at the source's position but did note a $2.9 \sigma$ source in the W2 filter that appeared to be fading (GCN 20400).

The BAT team performed a coincidence search but found nothing (GCN 20422).

Swift followed up another possible counterpart, iPTF17cw, but found no X-rays in $1.3 \mathrm{ks}$ worthy of observations (GCN 20473).

Seven GCN Circulars were sent by the Swift team in relation to this trigger: 20473, 20422, 20415, 20400, 20390, 20371.

\section{A.2. G270580/LVC 170120}

LVC detected an unmodeled burst trigger at 12:31:00 UT. The first PPST list was uploaded at 17:35 UT, and the first Swift observation occurred at T0+19.9 ks. Of the 159 planned tiles, 136 were observed, covering $1.1 \%$ of the raw (and 14\% of the convolved) LVC probability region. Three X-ray sources were detected: two known (rank 4) sources and one unlikely afterglow (rank 3 source).

Four GCN Circulars were sent by the Swift team. The highlights are as follows. GCN 20494: $18 \%$ of the LVC error region was covered by the $\mathrm{BAT}$ at the time of trigger, but no significant detection was found. GCN 20495: the abovementioned XRT sources were reported. GCN 20523: a report on the XRT follow-up of possible counterpart PS17yt; no source was detected in $3 \mathrm{ks}$ of observation. GCN 20536: a report on the UVOT follow-up of the same source, which was found at magnitude 20.9.

\section{A.3. G274296/LVC 170217}

LVC detected an unmodeled burst trigger at 06:05:53 UT. No tiling was performed, since the trigger did not meet our trigger criteria. The BAT covered $65.5 \%$ of the LVC error region at the time of trigger, but no significant detections were found (GCN 20744). 
Table 2

Catalog of X-Ray Sources Detected in the Follow-up Searches for O2 GW Triggers

\begin{tabular}{|c|c|c|c|c|c|c|c|c|c|c|}
\hline $\begin{array}{l}\text { GW } \\
\#\end{array}$ & Rank & $\begin{array}{l}\text { R.A./Decl. } \\
\text { (J2000) }\end{array}$ & $\begin{array}{c}\text { Err } \\
(\operatorname{arcsec})\end{array}$ & $\begin{array}{l}\text { Peak Rate } \\
\left(\text { counts s }^{-1}\right)\end{array}$ & $\begin{array}{c}\text { Peak Flux } \\
\left(\mathrm{erg} \mathrm{cm}^{-2} \mathrm{~s}^{-1}\right)\end{array}$ & $\begin{array}{l}\text { SIMBAD } \\
\text { Src Type }\end{array}$ & $\begin{array}{c}\text { Known } \\
\quad X\end{array}$ & $\begin{array}{l}\text { Near } \\
\text { Gal. }\end{array}$ & $\begin{array}{c}\text { Near } \\
\text { 2MASS }\end{array}$ & $\begin{array}{l}\text { Fading } \\
(\sigma)\end{array}$ \\
\hline 8 & 4 & $00^{\mathrm{h}} 00^{\mathrm{m}} 31^{\mathrm{s}} \cdot 64+68^{\circ} 15^{\prime} 00^{\prime \prime} 4$ & 4.9 & $0.028( \pm 0.009)$ & $1.2( \pm 0.4) \times 10^{-12}$ & Star & $\mathrm{Y}$ & 2 & 1 & 0 \\
\hline 8 & 4 & $01^{\mathrm{h}} 09^{\mathrm{m}} 44^{\mathrm{s}} .03+73^{\circ} 11^{\prime} 58^{\prime \prime} 5$ & 5.6 & $0.14( \pm 0.05)$ & $6.1( \pm 2.2) \times 10^{-12}$ & Seyfert 1 & $\mathrm{Y}$ & 1 & 1 & 0 \\
\hline 17 & 3 & $01^{\mathrm{h}} 20^{\mathrm{m}} 16^{\mathrm{s}} .99+12^{\circ} 03^{\prime} 20^{\prime \prime} 3$ & 6.1 & $0.013( \pm 0.005)$ & $5.7( \pm 2.1) \times 10^{-13}$ & Radio & $\mathrm{N}$ & 0 & 1 & 0 \\
\hline 17 & 3 & $01^{\mathrm{h}} 24^{\mathrm{m}} 24^{\mathrm{s}} \cdot 94+08^{\circ} 24^{\prime} 03^{\prime \prime} 1$ & 5.2 & $0.033( \pm 0.010)$ & $1.4( \pm 0.4) \times 10^{-12}$ & $\mathrm{~EB}^{*}$ WUMa & $\mathrm{N}$ & 1 & 1 & 0 \\
\hline 17 & 4 & $01^{\mathrm{h}} 24^{\mathrm{m}} 42^{\mathrm{s}} \cdot 22+08^{\circ} 51^{\prime} 24^{\prime \prime} 6$ & 4.5 & $0.046( \pm 0.012)$ & $2.0( \pm 0.5) \times 10^{-12}$ & Seyfert & $\mathrm{Y}$ & 1 & 1 & 0 \\
\hline 17 & 4 & $01^{\mathrm{h}} 39^{\mathrm{m}} 10^{\mathrm{s}} 99+34^{\circ} 33^{\prime} 40^{\prime \prime} 3$ & 6.6 & $0.068( \pm 0.028)$ & $2.9( \pm 1.2) \times 10^{-12}$ & $\mathrm{X}$ & $\mathrm{Y}$ & 0 & 1 & 0 \\
\hline 17 & 3 & $01^{\mathrm{h}} 40^{\mathrm{m}} 17^{\mathrm{s}} \cdot 34+23^{\circ} 27^{\prime} 21^{\prime \prime} 3$ & 6.4 & $0.020( \pm 0.008)$ & $9( \pm 3) \times 10^{-13}$ & QSO & $\mathrm{N}$ & 0 & 1 & 0 \\
\hline 17 & 4 & $01^{\mathrm{h}} 44^{\mathrm{m}} 47^{\mathrm{s}} \cdot 20+32^{\circ} 32^{\prime} 56^{\prime \prime} 3$ & 4.9 & $0.07( \pm 0.04)$ & $3.0( \pm 1.8) \times 10^{-12}$ & Unknown & Y & 0 & 1 & 0 \\
\hline 17 & 3 & $01^{\mathrm{h}} 45^{\mathrm{m}} 42^{\mathrm{s}} .52+32^{\circ} 43^{\prime} 39^{\prime \prime} 8$ & 6.8 & $0.028( \pm 0.011)$ & $1.2( \pm 0.5) \times 10^{-12}$ & Unknown & $\mathrm{N}$ & 0 & 1 & 0 \\
\hline 17 & 4 & $01^{\mathrm{h}} 46^{\mathrm{m}} 33^{\mathrm{s}} \cdot 86+33^{\circ} 17^{\prime} 08^{\prime \prime} 9$ & 6.3 & $0.12( \pm 0.05)$ & $5.2( \pm 2.2) \times 10^{-12}$ & BYDra & $\mathrm{Y}$ & 0 & 3 & 0 \\
\hline 17 & 2 & $01^{\mathrm{h}} 47^{\mathrm{m}} 09^{\mathrm{s}} \cdot 95+23^{\circ} 45^{\prime} 30^{\prime \prime} 5$ & 4.1 & $0.076( \pm 0.015)$ & $3.3( \pm 0.6) \times 10^{-12}$ & RS CVn & $\mathrm{Y}$ & 0 & 1 & 0 \\
\hline 17 & 3 & $01^{\mathrm{h}} 55^{\mathrm{m}} 19^{\mathrm{s}} .94+41^{\circ} 27^{\prime} 00^{\prime \prime} 3$ & 6.1 & $0.020( \pm 0.012)$ & $9( \pm 5) \times 10^{-13}$ & Unknown & $\mathrm{N}$ & 1 & 0 & 0 \\
\hline 17 & 4 & $01^{\mathrm{h}} 55^{\mathrm{m}} 35^{\mathrm{s}} \cdot 91+31^{\circ} 15^{\prime} 15^{\prime \prime} 2$ & 5.2 & $0.036( \pm 0.010)$ & $1.5( \pm 0.4) \times 10^{-12}$ & Seyfert 1 & $\mathrm{Y}$ & 2 & 1 & 0 \\
\hline 17 & 4 & $01^{\mathrm{h}} 57^{\mathrm{m}} 15^{\mathrm{s}} \cdot 37+31^{\circ} 54^{\prime} 14^{\prime \prime} 6$ & 5.7 & $0.027( \pm 0.010)$ & $1.1( \pm 0.4) \times 10^{-12}$ & Radio & $\mathrm{Y}$ & 0 & 1 & 0 \\
\hline 17 & 4 & $01^{\mathrm{h}} 57^{\mathrm{m}} 56^{\mathrm{s}} 31+40^{\circ} 24^{\prime} 17^{\prime \prime} 6$ & 6.8 & $0.022( \pm 0.009)$ & $10( \pm 4) \times 10^{-13}$ & Unknown & $\mathrm{Y}$ & 1 & 1 & 0 \\
\hline 17 & 3 & $01^{\mathrm{h}} 58^{\mathrm{m}} 48^{\mathrm{s}} \cdot 10+36^{\circ} 21^{\prime} 39^{\prime \prime} 6$ & 5.1 & $0.024( \pm 0.009)$ & $1.0( \pm 0.4) \times 10^{-12}$ & Unknown & $\mathrm{N}$ & 3 & 1 & 0 \\
\hline 17 & 3 & $02^{\mathrm{h}} 00^{\mathrm{m}} 38^{\mathrm{s}} \cdot 66+44^{\circ} 27^{\prime} 17^{\prime \prime} 8$ & 5.7 & $0.013( \pm 0.006)$ & $5.6( \pm 2.6) \times 10^{-13}$ & QSO & $\mathrm{N}$ & 0 & 0 & 0 \\
\hline 17 & 4 & $02^{\mathrm{h}} 01^{\mathrm{m}} 06^{\mathrm{s}} \cdot 90+44^{\circ} 08^{\prime} 42^{\prime \prime} 1$ & 4.7 & $0.062( \pm 0.014)$ & $2.7( \pm 0.6) \times 10^{-12}$ & Unknown & $\mathrm{Y}$ & 0 & 1 & 0 \\
\hline 17 & 3 & $02^{\mathrm{h}} 01^{\mathrm{m}} 09^{s} \cdot 81+44^{\circ} 10^{\prime} 16^{\prime \prime} 2$ & 5.8 & $0.018( \pm 0.008)$ & $8( \pm 3) \times 10^{-13}$ & (AGN; MQ) & $\mathrm{N}$ & 0 & 1 & 0 \\
\hline 17 & 3 & $02^{\mathrm{h}} 02^{\mathrm{m}} 01^{\mathrm{s}} .50+39^{\circ} 43^{\prime} 19^{\prime \prime} 5$ & 7.5 & $0.017( \pm 0.007)$ & $7( \pm 3) \times 10^{-13}$ & (AGN; MQ) & $\mathrm{N}$ & 1 & 1 & 0 \\
\hline 17 & 3 & $02^{\mathrm{h}} 03^{\mathrm{m}} 24^{\mathrm{s}} 54+39^{\circ} 51^{\prime} 19^{\prime \prime} 7$ & 7.9 & $0.04( \pm 0.03)$ & $1.8( \pm 1.3) \times 10^{-12}$ & Unknown & $\mathrm{N}$ & 0 & 1 & 1.2 \\
\hline 17 & 4 & $02^{\mathrm{h}} 12^{\mathrm{m}} 26^{\mathrm{s}} 58+52^{\circ} 09^{\prime} 51^{\prime \prime} 9$ & 5.4 & $0.047( \pm 0.013)$ & $2.0( \pm 0.6) \times 10^{-12}$ & AGN candidate & Y & 0 & 1 & 0 \\
\hline 17 & 4 & $02^{\mathrm{h}} 14^{\mathrm{m}} 17^{\mathrm{s}} .97+51^{\circ} 44^{\prime} 42^{\prime \prime} \cdot 9$ & 4.9 & $0.29( \pm 0.07)$ & $1.3( \pm 0.3) \times 10^{-11}$ & Unknown & $\mathrm{Y}$ & 5 & 0 & 0 \\
\hline 17 & 4 & $02^{\mathrm{h}} 18^{\mathrm{m}} 05^{\mathrm{s}} \cdot 26+39^{\circ} 17^{\prime} 44^{\prime \prime} 4$ & 7.9 & $0.045( \pm 0.012)$ & $2.0( \pm 0.5) \times 10^{-12}$ & $\mathrm{X}$ & $\mathrm{Y}$ & 4 & 1 & 0 \\
\hline 13 & 4 & $02^{\mathrm{h}} 18^{\mathrm{m}} 25^{\mathrm{s}} .40-50^{\circ} 13^{\prime} 29^{\prime \prime} 0$ & 5.7 & $0.017( \pm 0.006)$ & $7.5( \pm 2.7) \times 10^{-13}$ & $\mathrm{X}(\mathrm{AGN} ; \mathrm{MQ})$ & $\mathrm{Y}$ & 3 & 0 & 0 \\
\hline 13 & 4 & $02^{\mathrm{h}} 18^{\mathrm{m}} 30^{\mathrm{s}} \cdot 66-48^{\circ} 06^{\prime} 53^{\prime \prime} 1$ & 4.9 & $0.08( \pm 0.05)$ & $3.3( \pm 2.0) \times 10^{-12}$ & $\mathrm{X}$ & $\mathrm{Y}$ & 0 & 1 & 1.2 \\
\hline 13 & 3 & $02^{\mathrm{h}} 19^{\mathrm{m}} 28^{\mathrm{s}} \cdot 48-48^{\circ} 31^{\prime} 15^{\prime \prime} \cdot 2$ & 5.6 & $0.017( \pm 0.007)$ & $7( \pm 3) \times 10^{-13}$ & Galaxy & $\mathrm{N}$ & 2 & 1 & 0 \\
\hline 17 & 4 & $02^{\mathrm{h}} 19^{\mathrm{m}} 52^{\mathrm{s}} \cdot 91+43^{\circ} 55^{\prime} 18^{\prime \prime} 3$ & 6.8 & $0.018( \pm 0.010)$ & $8( \pm 4) \times 10^{-13}$ & Unknown & Y & 1 & 1 & 0 \\
\hline 17 & 3 & $02^{\mathrm{h}} 20^{\mathrm{m}} 14^{\mathrm{s}} .55+50^{\circ} 44^{\prime} 44^{\prime \prime} 1$ & 9.8 & $0.020( \pm 0.008)$ & $9( \pm 3) \times 10^{-13}$ & Unknown & $\mathrm{N}$ & 0 & 2 & 0 \\
\hline 13 & 3 & $02^{\mathrm{h}} 20^{\mathrm{m}} 23^{\mathrm{s}} \cdot 62-51^{\circ} 24^{\prime} 00^{\prime \prime} 8$ & 5.9 & $0.019( \pm 0.008)$ & $8( \pm 3) \times 10^{-13}$ & Unknown & $\mathrm{N}$ & 0 & 0 & 0 \\
\hline 17 & 4 & $02^{\mathrm{h}} 20^{\mathrm{m}} 35^{\mathrm{s}} \cdot 63+50^{\circ} 44^{\prime} 11^{\prime \prime} 6$ & 5.2 & $0.017( \pm 0.007)$ & $7( \pm 3) \times 10^{-13}$ & Unknown & $\mathrm{Y}$ & 0 & 1 & 0 \\
\hline 17 & 3 & $02^{\mathrm{h}} 21^{\mathrm{m}} 26^{\mathrm{s}} .96+51^{\circ} 26^{\prime} 11^{\prime \prime} 9$ & 5.1 & $0.013( \pm 0.005)$ & $5.4( \pm 2.0) \times 10^{-13}$ & Unknown & $\mathrm{N}$ & 0 & 1 & 0 \\
\hline 17 & 4 & $02^{\mathrm{h}} 22^{\mathrm{m}} 38^{\mathrm{s}} .72+43^{\circ} 02^{\prime} 09^{\prime \prime} 6$ & 4.3 & $0.14( \pm 0.06)$ & $5.8( \pm 2.4) \times 10^{-12}$ & Unknown & Y & 6 & 0 & 1.4 \\
\hline 17 & 3 & $02^{\mathrm{h}} 23^{\mathrm{m}} 05^{\mathrm{s}} \cdot 28+43^{\circ} 30^{\prime} 48^{\prime \prime} 4$ & 6.1 & $0.022( \pm 0.007)$ & $9( \pm 3) \times 10^{-13}$ & Unknown & $\mathrm{N}$ & 0 & 1 & 0 \\
\hline 13 & 3 & $02^{\mathrm{h}} 23^{\mathrm{m}} 29^{\mathrm{s}} .89-50^{\circ} 29^{\prime} 31^{\prime \prime} 7$ & 5.8 & $.01( \pm .004)$ & $4.1( \pm 1.8) \times 10^{-13}$ & Unknown & $\mathrm{N}$ & 0 & 0 & 0 \\
\hline 13 & 3 & $02^{\mathrm{h}} 24^{\mathrm{m}} 11^{\mathrm{s}} .57-49^{\circ} 53^{\prime} 04^{\prime \prime} 6$ & 5.1 & $0.035( \pm 0.013)$ & $1.5( \pm 0.5) \times 10^{-12}$ & Unknown & $\mathrm{N}$ & 0 & 1 & 0 \\
\hline 13 & 3 & $02^{\mathrm{h}} 25^{\mathrm{m}} 02^{\mathrm{s}} .96-53^{\circ} 52^{\prime} 59^{\prime \prime} 6$ & 5.1 & $0.045( \pm 0.011)$ & $1.9( \pm 0.5) \times 10^{-12}$ & Unknown & $\mathrm{N}$ & 1 & 1 & 0 \\
\hline 13 & 3 & $02^{\mathrm{h}} 25^{\mathrm{m}} 53^{\mathrm{s}} \cdot 44-48^{\circ} 26^{\prime} 28^{\prime \prime} \cdot 9$ & 6.1 & $0.022( \pm 0.009)$ & $9( \pm 4) \times 10^{-13}$ & Unknown & $\mathrm{N}$ & 0 & 1 & 0 \\
\hline 13 & 4 & $02^{\mathrm{h}} 26^{\mathrm{m}} 46^{\mathrm{s}} .93-50^{\circ} 37^{\prime} 56^{\prime \prime} 6$ & 6 & $0.014( \pm 0.007)$ & $6.0( \pm 2.8) \times 10^{-13}$ & $\mathrm{X}$ & $\mathrm{Y}$ & 0 & 0 & 0 \\
\hline 13 & 3 & $02^{\mathrm{h}} 27^{\mathrm{m}} 03^{\mathrm{s}} .80-49^{\circ} 48^{\prime} 08^{\prime \prime} 1$ & 6 & $0.022( \pm 0.008)$ & $9( \pm 3) \times 10^{-13}$ & Unknown & $\mathrm{N}$ & 0 & 0 & 0 \\
\hline 13 & 3 & $02^{\mathrm{h}} 28^{\mathrm{m}} 52^{\mathrm{s}} \cdot 21-49^{\circ} 21^{\prime} 36^{\prime \prime} 8$ & 6 & $0.05( \pm 0.04)$ & $2.0( \pm 1.5) \times 10^{-12}$ & (AGN; MQ) & $\mathrm{N}$ & 0 & 1 & 0 \\
\hline 13 & 4 & $02^{\mathrm{h}} 30^{\mathrm{m}} 00^{\mathrm{s}} \cdot 37-54^{\circ} 01^{\prime} 19^{\prime \prime} 8$ & 7.8 & $0.046( \pm 0.016)$ & $2.0( \pm 0.7) \times 10^{-12}$ & Unknown & $\mathrm{Y}$ & 0 & 0 & 0 \\
\hline 13 & 4 & $02^{\mathrm{h}} 30^{\mathrm{m}} 20^{\mathrm{s}} .85-54^{\circ} 15^{\prime} 08^{\prime \prime} 8$ & 5.2 & $0.034( \pm 0.012)$ & $1.5( \pm 0.5) \times 10^{-12}$ & Unknown & Y & 0 & 1 & 0 \\
\hline 13 & 4 & $02^{\mathrm{h}} 31^{\mathrm{m}} 31^{\mathrm{s}} .13-48^{\circ} 26^{\prime} 57^{\prime \prime} 4$ & 5 & $0.031( \pm 0.011)$ & $1.3( \pm 0.5) \times 10^{-12}$ & AGN candidate & $\mathrm{Y}$ & 0 & 1 & 0 \\
\hline 13 & 4 & $02^{\mathrm{h}} 34^{\mathrm{m}} 31^{\mathrm{s}} .24-46^{\circ} 31^{\prime} 59^{\prime \prime} 0$ & 6.3 & $0.11( \pm 0.05)$ & $4.9( \pm 2.3) \times 10^{-12}$ & Galaxy & $\mathrm{Y}$ & 0 & 1 & 0 \\
\hline 13 & 3 & $02^{\mathrm{h}} 35^{\mathrm{m}} 29^{\mathrm{s}} .57-50^{\circ} 21^{\prime} 21^{\prime \prime} 2$ & 6.3 & $0.05( \pm 0.04)$ & $2.3( \pm 1.7) \times 10^{-12}$ & Unknown & $\mathrm{N}$ & 1 & 2 & 1.4 \\
\hline 13 & 4 & $02^{\mathrm{h}} 38^{\mathrm{m}} 19^{\mathrm{s}} \cdot 17-52^{\circ} 11^{\prime} 33^{\prime \prime} \cdot 7$ & 3.6 & $1.06( \pm 0.07)$ & $4.5( \pm 0.3) \times 10^{-11}$ & Seyfert 1 & $\mathrm{Y}$ & 3 & 1 & 0 \\
\hline 13 & 4 & $02^{\mathrm{h}} 38^{\mathrm{m}} 20^{\mathrm{s}} \cdot 92-53^{\circ} 25^{\prime} 32^{\prime \prime} 5$ & 5.1 & $0.029( \pm 0.010)$ & $1.2( \pm 0.4) \times 10^{-12}$ & AGN candidate & $\mathrm{Y}$ & 1 & 1 & 0 \\
\hline 13 & 4 & $02^{\mathrm{h}} 42^{\mathrm{m}} 12^{\mathrm{s}} \cdot 18-54^{\circ} 57^{\prime} 13^{\prime \prime} 5$ & 5.7 & $0.022( \pm 0.008)$ & $9( \pm 4) \times 10^{-13}$ & Galaxy & Y & 0 & 1 & 0 \\
\hline 13 & 4 & $02^{\mathrm{h}} 42^{\mathrm{m}} 36^{\mathrm{s}} .78-55^{\circ} 06^{\prime} 35^{\prime \prime} 7$ & 4.8 & $0.035( \pm 0.010)$ & $1.5( \pm 0.4) \times 10^{-12}$ & Galaxy & $\mathrm{Y}$ & 2 & 1 & 0 \\
\hline 13 & 4 & $02^{\mathrm{h}} 45^{\mathrm{m}} 12^{\mathrm{s}} .97-46^{\circ} 27^{\prime} 55^{\prime \prime} 3$ & 6.7 & $0.017( \pm 0.008)$ & $7( \pm 3) \times 10^{-13}$ & Galaxy & $\mathrm{Y}$ & 5 & 2 & 0 \\
\hline 13 & 4 & $02^{\mathrm{h}} 45^{\mathrm{m}} 53^{\mathrm{s}} .78-44^{\circ} 59^{\prime} 38^{\prime \prime} 2$ & 5.4 & $0.08( \pm 0.05)$ & $3.5( \pm 2.1) \times 10^{-12}$ & QSO & $\mathrm{Y}$ & 0 & 1 & 0 \\
\hline 13 & 4 & $02^{\mathrm{h}} 51^{\mathrm{m}} 11^{\mathrm{s}} .70-47^{\circ} 53^{\prime} 14^{\prime \prime} 1$ & 5 & $0.047( \pm 0.011)$ & $2.0( \pm 0.5) \times 10^{-12}$ & RS CVn & $\mathrm{Y}$ & 0 & 1 & 0 \\
\hline 13 & 3 & $03^{\mathrm{h}} 09^{\mathrm{m}} 51^{\mathrm{s}} .89-43^{\circ} 31^{\prime} 15^{\prime \prime} 6$ & 6.8 & $0.011( \pm 0.005)$ & $4.8( \pm 2.1) \times 10^{-13}$ & (AGN; MQ) & $\mathrm{N}$ & 0 & 1 & 0 \\
\hline 13 & 4 & $03^{\mathrm{h}} 12^{\mathrm{m}} 25^{\mathrm{s}} \cdot 39-44^{\circ} 25^{\prime} 17^{\prime \prime} \cdot 3$ & 5.1 & $0.21( \pm 0.03)$ & $9.0( \pm 1.4) \times 10^{-12}$ & $\mathrm{PM}^{*}$ & Y & 0 & 1 & 0 \\
\hline 13 & 3 & $03^{\mathrm{h}} 13^{\mathrm{m}} 41^{\mathrm{s}} .96-44^{\circ} 41^{\prime} 08^{\prime \prime} 8$ & 6 & $0.020( \pm 0.008)$ & $9( \pm 3) \times 10^{-13}$ & Unknown & $\mathrm{N}$ & 0 & 0 & 0 \\
\hline 13 & 4 & $03^{\mathrm{h}} 13^{\mathrm{m}} 42^{\mathrm{s}} \cdot 35-41^{\circ} 59^{\prime} 39^{\prime \prime} .5$ & 4.9 & $0.035( \pm 0.011)$ & $1.5( \pm 0.5) \times 10^{-12}$ & AGN candidate & $\mathrm{Y}$ & 1 & 1 & 0 \\
\hline 13 & 4 & $03^{\mathrm{h}} 14^{\mathrm{m}} 51^{\mathrm{s}} \cdot 05-42^{\circ} 02^{\prime} 52^{\prime \prime} \cdot 7$ & 4.4 & $0.036( \pm 0.011)$ & $1.6( \pm 0.5) \times 10^{-12}$ & (AGN; MQ) & $\mathrm{Y}$ & 0 & 0 & 0 \\
\hline 13 & 4 & $03^{\mathrm{h}} 14^{\mathrm{m}} 55^{\mathrm{s}} \cdot 60-42^{\circ} 41^{\prime} 00^{\prime \prime} 8$ & 5 & $0.071( \pm 0.017)$ & $3.0( \pm 0.7) \times 10^{-12}$ & Seyfert 1 & Y & 2 & 1 & 0 \\
\hline 13 & 4 & $03^{\mathrm{h}} 16^{\mathrm{m}} 45^{\mathrm{s}} .04-42^{\circ} 31^{\prime} 30^{\prime \prime} 7$ & 5.7 & $0.050( \pm 0.016)$ & $2.1( \pm 0.7) \times 10^{-12}$ & $\mathrm{PM}^{*}$ & $\mathrm{Y}$ & 0 & 1 & 0 \\
\hline 13 & 3 & $03^{\mathrm{h}} 17^{\mathrm{m}} 53^{\mathrm{s}} .85-44^{\circ} 12^{\prime} 03^{\prime \prime} \cdot 9$ & 6.7 & $0.027( \pm 0.009)$ & $1.2( \pm 0.4) \times 10^{-12}$ & Unknown & $\mathrm{N}$ & 1 & 0 & 0 \\
\hline 13 & 3 & $03^{\mathrm{h}} 17^{\mathrm{m}} 56^{\mathrm{s}} .06-39^{\circ} 05^{\prime} 38^{\prime \prime} \cdot 2$ & 5 & $0.034( \pm 0.012)$ & $1.5( \pm 0.5) \times 10^{-12}$ & Unknown & $\mathrm{N}$ & 0 & 1 & 0 \\
\hline 13 & 4 & $03^{\mathrm{h}} 17^{\mathrm{m}} 56^{\mathrm{s}} .75-44^{\circ} 13^{\prime} 46^{\prime \prime} 7$ & 8.3 & $0.270( \pm 0.026)$ & $1.2( \pm 0.11) \times 10^{-11}$ & Unknown & Y & 2 & 2 & 0 \\
\hline
\end{tabular}


Table 2

(Continued)

\begin{tabular}{|c|c|c|c|c|c|c|c|c|c|c|}
\hline $\begin{array}{l}\text { GW } \\
\#\end{array}$ & Rank & $\begin{array}{l}\text { R.A./Decl. } \\
\text { (J2000) }\end{array}$ & $\begin{array}{c}\text { Err } \\
(\operatorname{arcsec})\end{array}$ & $\begin{array}{l}\text { Peak Rate } \\
\left(\text { counts s }^{-1}\right)\end{array}$ & $\begin{array}{c}\text { Peak Flux } \\
\left(\mathrm{erg} \mathrm{cm}^{-2} \mathrm{~s}^{-1}\right)\end{array}$ & $\begin{array}{l}\text { SIMBAD } \\
\text { Src Type }\end{array}$ & $\begin{array}{c}\text { Known } \\
\mathrm{X}\end{array}$ & $\begin{array}{l}\text { Near } \\
\text { Gal. }\end{array}$ & $\begin{array}{c}\text { Near } \\
\text { 2MASS }\end{array}$ & $\begin{array}{l}\text { Fading } \\
\qquad(\sigma)\end{array}$ \\
\hline 13 & 4 & $03^{\mathrm{h}} 17^{\mathrm{m}} 57^{\mathrm{s}} \cdot 19-44^{\circ} 15^{\prime} 39^{\prime \prime} 3$ & 5.8 & $0.176( \pm 0.023)$ & $7.5( \pm 1.0) \times 10^{-12}$ & QSO & $\mathrm{Y}$ & 2 & 2 & 0 \\
\hline 13 & 4 & $03^{\mathrm{h}} 17^{\mathrm{m}} 57^{\mathrm{s}} .53-44^{\circ} 14^{\prime} 19^{\prime \prime} 7$ & 5.2 & $0.180( \pm 0.023)$ & $7.7( \pm 1.0) \times 10^{-12}$ & QSO & $\mathrm{Y}$ & 2 & 2 & 0 \\
\hline 8 & 4 & $03^{\mathrm{h}} 17^{\mathrm{m}} 57^{\mathrm{s}} \cdot 54-44^{\circ} 14^{\prime} 15^{\prime \prime} 8$ & 4.7 & $0.12( \pm 0.06)$ & $5.0( \pm 2.6) \times 10^{-12}$ & EB*WUMa & $\mathrm{Y}$ & 0 & 1 & 1.3 \\
\hline 13 & 3 & $03^{\mathrm{h}} 18^{\mathrm{m}} 00^{\mathrm{s}} .85-44^{\circ} 12^{\prime} 06^{\prime \prime} 8$ & 8.4 & $0.037( \pm 0.011)$ & $1.6( \pm 0.5) \times 10^{-12}$ & Unknown & $\mathrm{N}$ & 1 & 0 & 0 \\
\hline 13 & 4 & $03^{\mathrm{h}} 18^{\mathrm{m}} 01^{\mathrm{s}} .64-44^{\circ} 13^{\prime} 43^{\prime \prime} 2$ & 8.5 & $0.041( \pm 0.011)$ & $1.8( \pm 0.5) \times 10^{-12}$ & Unknown & $\mathrm{Y}$ & 3 & 1 & 0 \\
\hline 13 & 4 & $03^{\mathrm{h}} 18^{\mathrm{m}} 01^{\mathrm{s}} \cdot 70-44^{\circ} 14^{\prime} 40^{\prime \prime} 1$ & 9.6 & $0.070( \pm 0.014)$ & $3.0( \pm 0.6) \times 10^{-12}$ & Unknown & $\mathrm{Y}$ & 4 & 0 & 0 \\
\hline 13 & 3 & $03^{\mathrm{h}} 18^{\mathrm{m}} 09^{\mathrm{s}} .58-44^{\circ} 11^{\prime} 44^{\prime \prime} 5$ & 6.2 & $0.016( \pm 0.007)$ & $7( \pm 3) \times 10^{-13}$ & Unknown & $\mathrm{N}$ & 0 & 0 & 0 \\
\hline 13 & 4 & $03^{\mathrm{h}} 32^{\mathrm{m}} 49^{\mathrm{s}} .09-26^{\circ} 02^{\prime} 45^{\prime \prime} 0$ & 6.8 & $0.021( \pm 0.008)$ & $9( \pm 3) \times 10^{-13}$ & QSO & $\mathrm{Y}$ & 0 & 0 & 0 \\
\hline 17 & 3 & $03^{\mathrm{h}} 49^{\mathrm{m}} 43^{\mathrm{s}} \cdot 13+75^{\circ} 16^{\prime} 07^{\prime \prime} 6$ & 5.9 & $0.017( \pm 0.007)$ & $7( \pm 3) \times 10^{-13}$ & Unknown & $\mathrm{N}$ & 2 & 1 & 0 \\
\hline 17 & 4 & $04^{\mathrm{h}} 57^{\mathrm{m}} 53^{\stackrel{\mathrm{s}}{2} 20+80^{\circ} 06^{\prime} 50^{\prime \prime} 9}$ & 8.4 & $0.021( \pm 0.008)$ & $9( \pm 3) \times 10^{-13}$ & Unknown & $\mathrm{Y}$ & 1 & 1 & 0 \\
\hline 5 & 4 & $07^{\mathrm{h}} 20^{\mathrm{m}} 28^{\mathrm{s}} \cdot 70+71^{\circ} 32^{\prime} 35^{\prime \prime} 0$ & 5.7 & $0.18( \pm 0.07)$ & $8( \pm 3) \times 10^{-12}$ & Unknown & $\mathrm{Y}$ & 2 & 0 & 0 \\
\hline 4 & 4 & $08^{\mathrm{h}} 52^{\mathrm{m}} 20^{\mathrm{s}} \cdot 14+47^{\circ} 34^{\prime} 57^{\prime \prime} 8$ & 5.4 & $0.18( \pm 0.07)$ & $7.9( \pm 2.9) \times 10^{-12}$ & QSO & $\mathrm{Y}$ & 0 & 0 & 0 \\
\hline 4 & 4 & $08^{\mathrm{h}} 53^{\mathrm{m}} 46^{\mathrm{s}} .02+47^{\circ} 18^{\prime} 42^{\prime \prime} 0$ & 5 & $0.21( \pm 0.07)$ & $9.1( \pm 2.9) \times 10^{-12}$ & $\operatorname{Rot}^{*}$ & $\mathrm{Y}$ & 0 & 1 & 0 \\
\hline 8 & 2 & $09^{\mathrm{h}} 29^{\mathrm{m}} 46^{\mathrm{s}} \cdot 62+02^{\circ} 03^{\prime} 47^{\prime \prime} 8$ & 6 & $0.05( \pm 0.04)$ & $2.3( \pm 1.8) \times 10^{-12}$ & LINER & $\mathrm{N}$ & 5 & 1 & 4.1 \\
\hline 8 & 4 & $09^{\mathrm{h}} 47^{\mathrm{m}} 02^{\mathrm{s}} .28-05^{\circ} 56^{\prime} 50^{\prime \prime} 6$ & 5.7 & $0.017( \pm 0.009)$ & $7( \pm 4) \times 10^{-13}$ & Galaxy & $\mathrm{Y}$ & 1 & 1 & 0 \\
\hline 8 & 4 & $09^{\mathrm{h}} 57^{\mathrm{m}} 17^{\mathrm{s}} .97-13^{\circ} 50^{\prime} 00^{\prime \prime} 2$ & 6.1 & $0.025( \pm 0.015)$ & $1.1( \pm 0.6) \times 10^{-12}$ & QSO & $\mathrm{Y}$ & 2 & 0 & 0 \\
\hline 8 & 3 & $09^{\mathrm{h}} 58^{\mathrm{m}} 13^{\mathrm{s}} \cdot 62-05^{\circ} 24^{\prime} 30^{\prime \prime} 6$ & 6 & $0.019( \pm 0.008)$ & $8( \pm 3) \times 10^{-13}$ & Galaxy & $\mathrm{N}$ & 0 & 1 & 0 \\
\hline 8 & 4 & $09^{\mathrm{h}} 58^{\mathrm{m}} 33^{\mathrm{s}} \cdot 58-05^{\circ} 21^{\prime} 37^{\prime \prime} 9$ & 4.7 & $0.07( \pm 0.04)$ & $3.0( \pm 1.8) \times 10^{-12}$ & Unknown & Y & 0 & 1 & 0 \\
\hline 8 & 3 & $10^{\mathrm{h}} 02^{\mathrm{m}} 45^{\mathrm{s}} .70-16^{\circ} 11^{\prime} 48^{\prime \prime} 5$ & 5.6 & $0.020( \pm 0.008)$ & $9( \pm 4) \times 10^{-13}$ & (AGN; MQ) & $\mathrm{N}$ & 0 & 0 & 0 \\
\hline 8 & 4 & $10^{\mathrm{h}} 03^{\mathrm{m}} 41^{\mathrm{s}} .72-15^{\circ} 08^{\prime} 01^{\prime \prime} 3$ & 5.6 & $0.07( \pm 0.05)$ & $3.1( \pm 2.4) \times 10^{-12}$ & QSO & $\mathrm{Y}$ & 2 & 1 & 1.1 \\
\hline 8 & 4 & $10^{\mathrm{h}} 08^{\mathrm{m}} 03^{\mathrm{s}} .01-14^{\circ} 59^{\prime} 00^{\prime \prime} \cdot 9$ & 4.6 & $0.07( \pm 0.04)$ & $3.0( \pm 1.8) \times 10^{-12}$ & Seyfert 1 & $\mathrm{Y}$ & 1 & 2 & 0 \\
\hline 8 & 4 & $10^{\mathrm{h}} 11^{\mathrm{m}} 59^{\mathrm{s}} \cdot 30-16^{\circ} 36^{\prime} 31^{\prime \prime} 6$ & 5 & $0.039( \pm 0.011)$ & $1.7( \pm 0.5) \times 10^{-12}$ & AGN & $\mathrm{Y}$ & 1 & 1 & 0 \\
\hline 8 & 4 & $10^{\mathrm{h}} 13^{\mathrm{m}} 05^{\mathrm{s}} .43-16^{\circ} 41^{\prime} 21^{\prime \prime} 0$ & 6.7 & $0.016( \pm 0.008)$ & $7( \pm 3) \times 10^{-13}$ & $\mathrm{X}$ & $\mathrm{Y}$ & 0 & 0 & 0 \\
\hline 8 & 4 & $10^{\mathrm{h}} 15^{\mathrm{m}} 03^{\mathrm{s}} .40-16^{\circ} 52^{\prime} 10^{\prime \prime} 4$ & 4.8 & $0.034( \pm 0.011)$ & $1.4( \pm 0.5) \times 10^{-12}$ & QSO & $\mathrm{Y}$ & 0 & 1 & 0 \\
\hline 8 & 4 & $10^{\mathrm{h}} 15^{\mathrm{m}} 56^{\mathrm{s}} .13-20^{\circ} 02^{\prime} 28^{\prime \prime} 3$ & 5.6 & $0.17( \pm 0.06)$ & $7.1( \pm 2.6) \times 10^{-12}$ & Seyfert 1 & $\mathrm{Y}$ & 2 & 1 & 0 \\
\hline 8 & 2 & $10^{\mathrm{h}} 18^{\mathrm{m}} 47^{\mathrm{s}} .53-18^{\circ} 32^{\prime} 39^{\prime \prime} \cdot 2$ & 5.1 & $0.05( \pm 0.03)$ & $2.0( \pm 1.5) \times 10^{-12}$ & Unknown & $\mathrm{N}$ & 0 & 0 & 4.3 \\
\hline 8 & 4 & $10^{\mathrm{h}} 23^{\mathrm{m}} 34^{\mathrm{s}} .73-19^{\circ} 32^{\prime} 35^{\prime \prime} 6$ & 5.2 & $0.05( \pm 0.04)$ & $2.3( \pm 1.7) \times 10^{-12}$ & Star & $\mathrm{Y}$ & 2 & 1 & 1.3 \\
\hline 8 & 4 & $10^{\mathrm{h}} 24^{\mathrm{m}} 52^{\mathrm{s}} .73-19^{\circ} 56^{\prime} 14^{\prime \prime} 2$ & 6.8 & $0.018( \pm 0.008)$ & $8( \pm 3) \times 10^{-13}$ & $\mathrm{X}$ & $\mathrm{Y}$ & 0 & 1 & 0 \\
\hline 8 & 4 & $10^{\mathrm{h}} 50^{\mathrm{m}} 57^{\mathrm{s}} .19-28^{\circ} 50^{\prime} 00^{\prime \prime} 5$ & 5.1 & $0.018( \pm 0.008)$ & $8( \pm 4) \times 10^{-13}$ & Star & $\mathrm{Y}$ & 0 & 1 & 0 \\
\hline 8 & 4 & $11^{\mathrm{h}} 18^{\mathrm{m}} 15^{\mathrm{s}} \cdot 20-32^{\circ} 48^{\prime} 12^{\prime \prime} 2$ & 6.5 & $0.020( \pm 0.008)$ & $9( \pm 3) \times 10^{-13}$ & Unknown & $\mathrm{Y}$ & 0 & 0 & 0 \\
\hline 8 & 4 & $11^{\mathrm{h}} 19^{\mathrm{m}} 45^{\mathrm{s}} .86-34^{\circ} 35^{\prime} 51^{\prime \prime} 5$ & 6.8 & $0.019( \pm 0.009)$ & $8( \pm 4) \times 10^{-13}$ & $\mathrm{X}$ & $\mathrm{Y}$ & 1 & 1 & 0 \\
\hline 8 & 4 & $11^{\mathrm{h}} 36^{\mathrm{m}} 16^{\mathrm{s}} \cdot 20-38^{\circ} 02^{\prime} 08^{\prime \prime} \cdot 2$ & 6.8 & $0.09( \pm 0.04)$ & $3.9( \pm 1.5) \times 10^{-12}$ & RS CVn & $\mathrm{Y}$ & 1 & 2 & 0 \\
\hline 8 & 4 & $11^{\mathrm{h}} 39^{\mathrm{m}} 01^{\mathrm{s}} \cdot 66-37^{\circ} 44^{\prime} 17^{\prime \prime} \cdot 2$ & 4.2 & $0.98( \pm 0.19)$ & $4.2( \pm 0.8) \times 10^{-11}$ & Seyfert 1 & $\mathrm{Y}$ & 0 & 1 & 1.1 \\
\hline 13 & 4 & $11^{\mathrm{h}} 42^{\mathrm{m}} 47^{\mathrm{s}} .84-35^{\circ} 48^{\prime} 57^{\prime \prime} 1$ & 7.1 & $0.049( \pm 0.012)$ & $2.1( \pm 0.5) \times 10^{-12}$ & Unknown & $\mathrm{Y}$ & 3 & 0 & 0 \\
\hline 14 & 4 & $11^{\mathrm{h}} 47^{\mathrm{m}} 56^{\mathrm{s}} \cdot 31-38^{\circ} 58^{\prime} 13^{\prime \prime} 2$ & 6.4 & $0.09( \pm 0.06)$ & $4.1( \pm 2.4) \times 10^{-12}$ & Unknown & $\mathrm{Y}$ & 0 & 1 & 0 \\
\hline 8 & 4 & $12^{\mathrm{h}} 10^{\mathrm{m}} 04^{\mathrm{s}} .18-46^{\circ} 36^{\prime} 24^{\prime \prime} 0$ & 4.3 & $0.113( \pm 0.020)$ & $4.9( \pm 0.8) \times 10^{-12}$ & Seyfert 2 & $\mathrm{Y}$ & 1 & 1 & 0 \\
\hline 8 & 4 & $12^{\mathrm{h}} 11^{\mathrm{m}} 15^{\mathrm{s}} \cdot 32-46^{\circ} 41^{\prime} 31^{\prime \prime} 8$ & 7 & $0.022( \pm 0.009)$ & $9( \pm 4) \times 10^{-13}$ & Unknown & $\mathrm{Y}$ & 5 & 1 & 0 \\
\hline 8 & 3 & $12^{\mathrm{h}} 23^{\mathrm{m}} 18^{\mathrm{s}} .14-44^{\circ} 37^{\prime} 31^{\prime \prime} 4$ & 6.4 & $0.031( \pm 0.012)$ & $1.3( \pm 0.5) \times 10^{-12}$ & Unknown & $\mathrm{N}$ & 0 & 1 & 1.1 \\
\hline 14 & 4 & $12^{\mathrm{h}} 45^{\mathrm{m}} 57^{\mathrm{s}} .78-12^{\circ} 51^{\prime} 18^{\prime \prime} 0$ & 7.1 & $0.066( \pm 0.028)$ & $2.8( \pm 1.2) \times 10^{-12}$ & Galaxy & Y & 0 & 1 & 0 \\
\hline 14 & 4 & $12^{\mathrm{h}} 49^{\mathrm{m}} 10^{\mathrm{s}} .81-11^{\circ} 49^{\prime} 24^{\prime \prime} 3$ & 5.9 & $0.08( \pm 0.03)$ & $3.4( \pm 1.3) \times 10^{-12}$ & Galaxy & $\mathrm{Y}$ & 4 & 1 & 0 \\
\hline 14 & 4 & $12^{\mathrm{h}} 52^{\mathrm{m}} 12^{\mathrm{s}} \cdot 65-13^{\circ} 24^{\prime} 52^{\prime \prime} 6$ & 4.4 & $0.31( \pm 0.06)$ & $1.3( \pm 0.2) \times 10^{-11}$ & $\begin{array}{c}\text { Radio } \\
\text { (AGN; MQ) }\end{array}$ & $\mathrm{Y}$ & 4 & 1 & 0 \\
\hline 14 & 4 & $13^{\mathrm{h}} 00^{\mathrm{m}} 54^{\mathrm{s}} .83-21^{\circ} 34^{\prime} 20^{\prime \prime} 6$ & 5.7 & $0.054( \pm 0.025)$ & $2.3( \pm 1.1) \times 10^{-12}$ & Unknown & $\mathrm{Y}$ & 0 & 1 & 0 \\
\hline 14 & 4 & $13^{\mathrm{h}} 12^{\mathrm{m}} 31^{\mathrm{s}} .88-21^{\circ} 56^{\prime} 18^{\prime \prime} \cdot 9$ & 5.8 & $0.070( \pm 0.029)$ & $3.0( \pm 1.3) \times 10^{-12}$ & BL Lac & $\mathrm{Y}$ & 0 & 1 & 0 \\
\hline 14 & 3 & $13^{\mathrm{h}} 25^{\mathrm{m}} 07^{\mathrm{s}} .31-32^{\circ} 38^{\prime} 14^{\prime \prime} 4$ & 5.5 & $0.14( \pm 0.04)$ & $6.2( \pm 1.8) \times 10^{-12}$ & Radio & $\mathrm{N}$ & 0 & 1 & 0 \\
\hline 14 & 4 & $13^{\mathrm{h}} 26^{\mathrm{m}} 16^{\mathrm{s}} 69-29^{\circ} 05^{\prime} 13^{\prime \prime} 0$ & 5.3 & $0.08( \pm 0.03)$ & $3.6( \pm 1.4) \times 10^{-12}$ & $\operatorname{Rot}^{*}$ & $\mathrm{Y}$ & 3 & 1 & 0 \\
\hline 14 & 4 & $13^{\mathrm{h}} 35^{\mathrm{m}} 53^{\mathrm{s}} .88-34^{\circ} 17^{\prime} 41^{\prime \prime} 2$ & 3.8 & $1.78( \pm 0.20)$ & $7.7( \pm 0.8) \times 10^{-11}$ & Seyfert 1 & $\mathrm{Y}$ & 8 & 1 & 0 \\
\hline 14 & 4 & $13^{\mathrm{h}} 36^{\mathrm{m}} 39^{\mathrm{s}} \cdot 33-33^{\circ} 57^{\prime} 58^{\prime \prime} 0$ & 6.3 & $0.07( \pm 0.03)$ & $3.1( \pm 1.4) \times 10^{-12}$ & AGN & $\mathrm{Y}$ & 7 & 1 & 1.2 \\
\hline 14 & 4 & $13^{\mathrm{h}} 38^{\mathrm{m}} 23^{\mathrm{s}} .70-36^{\circ} 14^{\prime} 01^{\prime \prime} 6$ & 4.7 & $0.17( \pm 0.05)$ & $7.3( \pm 2.0) \times 10^{-12}$ & Unknown & $\mathrm{Y}$ & 3 & 0 & 0 \\
\hline 14 & 4 & $14^{\mathrm{h}} 10^{\mathrm{m}} 34^{\mathrm{s}} .05-52^{\circ} 19^{\prime} 06^{\prime \prime} 9$ & 6.1 & $0.11( \pm 0.03)$ & $4.8( \pm 1.5) \times 10^{-12}$ & GinGroup & $\mathrm{Y}$ & 4 & 1 & 0 \\
\hline 8 & 4 & $14^{\mathrm{h}} 35^{\mathrm{m}} 47^{\mathrm{s}} .28-52^{\circ} 40^{\prime} 50^{\prime \prime} \cdot 9$ & 5.9 & $0.19( \pm 0.07)$ & $8.0( \pm 2.9) \times 10^{-12}$ & Star & $\mathrm{Y}$ & 1 & 1 & 0 \\
\hline 8 & 3 & $14^{\mathrm{h}} 45^{\mathrm{m}} 41^{\mathrm{s}} 86-49^{\circ} 22^{\prime} 28^{\prime \prime} 6$ & 6.7 & $0.019( \pm 0.008)$ & $8( \pm 3) \times 10^{-13}$ & Unknown & $\mathrm{N}$ & 1 & 0 & 0 \\
\hline 8 & 4 & $15^{\mathrm{h}} 10^{\mathrm{m}} 05^{\mathrm{s}} .74-50^{\circ} 51^{\prime} 33^{\prime \prime} 7$ & 5.9 & $0.09( \pm 0.05)$ & $4.0( \pm 2.1) \times 10^{-12}$ & Unknown & $\mathrm{Y}$ & 2 & 4 & 2 \\
\hline 8 & 3 & $15^{\mathrm{h}} 23^{\mathrm{m}} 17^{\mathrm{s}} .80-50^{\circ} 27^{\prime} 03^{\prime \prime} 6$ & 6.2 & $0.049( \pm 0.011)$ & $2.1( \pm 0.5) \times 10^{-12}$ & Unknown & $\mathrm{N}$ & 0 & 3 & 0 \\
\hline 17 & 4 & $16^{\mathrm{h}} 27^{\mathrm{m}} 04^{\mathrm{s}} \cdot 41+14^{\circ} 21^{\prime} 23^{\prime \prime} 3$ & 5.9 & $0.08( \pm 0.05)$ & $3.4( \pm 2.1) \times 10^{-12}$ & Seyfert 1 & $\mathrm{Y}$ & 2 & 1 & 0 \\
\hline 17 & 4 & $16^{\mathrm{h}} 44^{\mathrm{m}} 39^{\mathrm{s}} .08-01^{\circ} 51^{\prime} 55^{\prime \prime} 5$ & 4.7 & $0.037( \pm 0.012)$ & $1.6( \pm 0.5) \times 10^{-12}$ & Unknown & $\mathrm{Y}$ & 1 & 0 & 0 \\
\hline 17 & 4 & $16^{\mathrm{h}} 58^{\mathrm{m}} 41^{\mathrm{s}} \cdot 90-03^{\circ} 14^{\prime} 15^{\prime \prime} 2$ & 5.8 & $0.027( \pm 0.010)$ & $1.1( \pm 0.4) \times 10^{-12}$ & AGN candidate & $\mathrm{Y}$ & 1 & 2 & 0 \\
\hline 17 & 3 & $18^{\mathrm{h}} 22^{\mathrm{m}} 20^{\mathrm{s}} 87-24^{\circ} 30^{\prime} 04^{\prime \prime} 3$ & 6.9 & $0.022( \pm 0.009)$ & $10( \pm 4) \times 10^{-13}$ & Radio & $\mathrm{N}$ & 0 & 3 & 0 \\
\hline 17 & 3 & $18^{\mathrm{h}} 28^{\mathrm{m}} 06^{\mathrm{s}} \cdot 37-26^{\circ} 45^{\prime} 24^{\prime \prime} 0$ & 6 & $0.025( \pm 0.010)$ & $1.1( \pm 0.4) \times 10^{-12}$ & $* *$ & $\mathrm{~N}$ & 0 & 1 & 0 \\
\hline 5 & 4 & $18^{\mathrm{h}} 40^{\mathrm{m}} 38^{s} \cdot 99-77^{\circ} 09^{\prime} 32^{\prime \prime} 7$ & 5.1 & $0.21( \pm 0.06)$ & $8.9( \pm 2.6) \times 10^{-12}$ & Radio (cm) & $\mathrm{Y}$ & 10 & 1 & 0 \\
\hline 17 & 3 & $18^{\mathrm{h}} 41^{\mathrm{m}} 36^{\mathrm{s}} .88-26^{\circ} 54^{\prime} 18^{\prime \prime} \cdot 7$ & 6.5 & $0.013( \pm 0.005)$ & $5.5( \pm 2.0) \times 10^{-13}$ & Unknown & $\mathrm{N}$ & 0 & 2 & 0 \\
\hline 17 & 3 & $18^{\mathrm{h}} 41^{\mathrm{m}} 42^{\mathrm{s}} \cdot 47-31^{\circ} 11^{\prime} 33^{\prime \prime} 8$ & 4.6 & $0.050( \pm 0.012)$ & $2.1( \pm 0.5) \times 10^{-12}$ & Unknown & $\mathrm{N}$ & 0 & 1 & 0 \\
\hline 17 & 3 & $18^{\mathrm{h}} 41^{\mathrm{m}} 47^{\mathrm{s}} .10-31^{\circ} 10^{\prime} 05^{\prime \prime} 7$ & 5.5 & $0.029( \pm 0.010)$ & $1.2( \pm 0.4) \times 10^{-12}$ & Unknown & $\mathrm{N}$ & 0 & 1 & 0 \\
\hline
\end{tabular}


Table 2

(Continued)

\begin{tabular}{|c|c|c|c|c|c|c|c|c|c|c|}
\hline $\begin{array}{l}\text { GW } \\
\#\end{array}$ & Rank & $\begin{array}{l}\text { R.A./Decl. } \\
\text { (J2000) }\end{array}$ & $\begin{array}{c}\text { Err } \\
(\operatorname{arcsec})\end{array}$ & $\begin{array}{l}\text { Peak Rate } \\
\left(\text { counts s }{ }^{-1}\right)\end{array}$ & $\begin{array}{c}\text { Peak Flux } \\
\left(\mathrm{erg} \mathrm{cm}^{-2} \mathrm{~s}^{-1}\right)\end{array}$ & $\begin{array}{l}\text { SIMBAD } \\
\text { Src Type }\end{array}$ & $\begin{array}{c}\text { Known } \\
X\end{array}$ & $\begin{array}{l}\text { Near } \\
\text { Gal. }\end{array}$ & $\begin{array}{l}\text { Near } \\
\text { 2MASS }\end{array}$ & $\begin{array}{l}\text { Fading } \\
\qquad(\sigma)\end{array}$ \\
\hline 7 & 4 & $19^{\mathrm{h}} 59^{\mathrm{m}} 28^{\mathrm{s}} \cdot 64+40^{\circ} 44^{\prime} 13^{\prime \prime} 0$ & 8.2 & $2.7( \pm 0.5)$ & $1.16( \pm 0.23) \times 10^{-10}$ & Seyfert 2 & Y & 4 & 2 & 0 \\
\hline 17 & 4 & $20^{\mathrm{h}} 33^{\mathrm{m}} 00^{\mathrm{s}} 32-34^{\circ} 40^{\prime} 08^{\prime \prime} \cdot 9$ & 6 & $0.017( \pm 0.006)$ & $7.5( \pm 2.5) \times 10^{-13}$ & Unknown & $\mathrm{Y}$ & 0 & 1 & 0 \\
\hline 17 & 4 & $20^{\mathrm{h}} 36^{\mathrm{m}} 08^{\mathrm{s}} \cdot 50-36^{\circ} 07^{\prime} 09^{\prime \prime} 5$ & 4.2 & $0.091( \pm 0.016)$ & $3.9( \pm 0.7) \times 10^{-12}$ & $\operatorname{Rot}^{*}$ & Y & 2 & 1 & 0 \\
\hline 8 & 3 & $20^{\mathrm{h}} 56^{\mathrm{m}} 28^{\mathrm{s}} .43+30^{\circ} 45^{\prime} 17^{\prime \prime} 6$ & 6.1 & $0.025( \pm 0.012)$ & $1.1( \pm 0.5) \times 10^{-12}$ & Unknown & $\mathrm{N}$ & 0 & 0 & 1.7 \\
\hline 8 & 4 & $20^{\mathrm{h}} 58^{\mathrm{m}} 12^{\mathrm{s}} \cdot 30+30^{\circ} 04^{\prime} 36^{\prime \prime} \cdot 9$ & 4.3 & $0.100( \pm 0.017)$ & $4.3( \pm 0.7) \times 10^{-12}$ & Seyfert 1 & $\mathrm{Y}$ & 1 & 2 & 0 \\
\hline 8 & 4 & $20^{\mathrm{h}} 58^{\mathrm{m}} 52^{\mathrm{s}} .39+31^{\circ} 30^{\prime} 12^{\prime \prime} .2$ & 5.8 & $0.017( \pm 0.006)$ & $7.5( \pm 2.7) \times 10^{-13}$ & Unknown & $\mathrm{Y}$ & 0 & 2 & 0 \\
\hline 8 & 3 & $21^{\mathrm{h}} 11^{\mathrm{m}} 42^{\mathrm{s}} .05+32^{\circ} 59^{\prime} 25^{\prime \prime} \cdot 1$ & 7.2 & $0.019( \pm 0.008)$ & $8( \pm 4) \times 10^{-13}$ & Radio & $\mathrm{N}$ & 1 & 3 & 0 \\
\hline 8 & 3 & $21^{\mathrm{h}} 11^{\mathrm{m}} 55^{\mathrm{s}} .13+32^{\circ} 44^{\prime} 46^{\prime \prime} 9$ & 6.4 & $0.031( \pm 0.011)$ & $1.3( \pm 0.5) \times 10^{-12}$ & Unknown & $\mathrm{N}$ & 0 & 1 & 1.3 \\
\hline 8 & 4 & $21^{\mathrm{h}} 13^{\mathrm{m}} 44^{\mathrm{s}} .93+35^{\circ} 31^{\prime} 52^{\prime \prime} 4$ & 6.1 & $0.027( \pm 0.010)$ & $1.1( \pm 0.4) \times 10^{-12}$ & Unknown & $\mathrm{Y}$ & 0 & 1 & 0 \\
\hline 8 & 3 & $21^{\mathrm{h}} 17^{\mathrm{m}} 11^{\mathrm{s}} .49+36^{\circ} 04^{\prime} 31^{\prime \prime} \cdot 9$ & 5.6 & $0.023( \pm 0.009)$ & $10( \pm 4) \times 10^{-13}$ & Star & $\mathrm{N}$ & 0 & 2 & 0 \\
\hline 8 & 4 & $21^{\mathrm{h}} 21^{\mathrm{m}} 01^{\mathrm{s}} .16+40^{\circ} 20^{\prime} 36^{\prime \prime} 2$ & 4.5 & $0.08( \pm 0.04)$ & $3.3( \pm 1.5) \times 10^{-12}$ & $\mathrm{SB}^{*}$ & Y & 1 & 1 & 0 \\
\hline 8 & 3 & $21^{\mathrm{h}} 24^{\mathrm{m}} 30^{\mathrm{s}} .89+40^{\circ} 15^{\prime} 58^{\prime \prime} \cdot 4$ & 6.2 & $0.020( \pm 0.005)$ & $8.6( \pm 2.1) \times 10^{-13}$ & Unknown & $\mathrm{N}$ & 1 & 1 & 0 \\
\hline 8 & 4 & $21^{\mathrm{h}} 27^{\mathrm{m}} 14^{\mathrm{s}} \cdot 46+39^{\circ} 12^{\prime} 34^{\prime \prime} 5$ & 6 & $0.016( \pm 0.007)$ & $6.8( \pm 2.9) \times 10^{-13}$ & Star & $\mathrm{Y}$ & 2 & 1 & 0 \\
\hline 8 & 3 & $21^{\mathrm{h}} 33^{\mathrm{m}} 14^{\mathrm{s}} \cdot 37+39^{\circ} 41^{\prime} 07^{\prime \prime} 0$ & 5.5 & $0.06( \pm 0.04)$ & $2.8( \pm 1.7) \times 10^{-12}$ & Unknown & $\mathrm{N}$ & 0 & 1 & 1.6 \\
\hline 8 & 3 & $21^{\mathrm{h}} 39^{\mathrm{m}} 54^{\mathrm{s}} .85+44^{\circ} 45^{\prime} 51^{\prime \prime} 1$ & 4.9 & $0.11( \pm 0.05)$ & $4.7( \pm 2.2) \times 10^{-12}$ & Unknown & $\mathrm{N}$ & 1 & 1 & 1.7 \\
\hline 8 & 4 & $21^{\mathrm{h}} 42^{\mathrm{m}} 43^{\mathrm{s}} .28+43^{\circ} 35^{\prime} 12^{\prime \prime} 3$ & 4.2 & $2.7( \pm 0.4)$ & $1.17( \pm 0.18) \times 10^{-10}$ & Dwarf nova & $\mathrm{Y}$ & 10 & 1 & 0 \\
\hline 8 & 4 & $21^{\mathrm{h}} 53^{\mathrm{m}} 15^{\mathrm{s}} .93+47^{\circ} 43^{\prime} 48^{\prime \prime} 6$ & 5.2 & $0.023( \pm 0.010)$ & $10( \pm 4) \times 10^{-13}$ & Star & Y & 0 & 1 & 0 \\
\hline 8 & 4 & $22^{\mathrm{h}} 04^{\mathrm{m}} 56^{\mathrm{s}} .63+47^{\circ} 14^{\prime} 08^{\prime \prime} .4$ & 5.5 & $0.15( \pm 0.06)$ & $6.6( \pm 2.4) \times 10^{-12}$ & RS CVn & $\mathrm{Y}$ & 0 & 1 & 0 \\
\hline 8 & 3 & $22^{\mathrm{h}} 07^{\mathrm{m}} 29^{\mathrm{s}} \cdot 62+49^{\circ} 31^{\prime} 01^{\prime \prime} 1$ & 6.2 & $0.024( \pm 0.008)$ & $1.0( \pm 0.3) \times 10^{-12}$ & Unknown & $\mathrm{N}$ & 2 & 1 & 0 \\
\hline 8 & 4 & $22^{\mathrm{h}} 07^{\mathrm{m}} 47^{\mathrm{s}} .03+49^{\circ} 31^{\prime} 44^{\prime \prime} 3$ & 5.3 & $0.021( \pm 0.008)$ & $9( \pm 3) \times 10^{-13}$ & Star & $\mathrm{Y}$ & 3 & 1 & 1 \\
\hline 8 & 3 & $22^{\mathrm{h}} 08^{\mathrm{m}} 13^{\mathrm{s}} .89+53^{\circ} 06^{\prime} 51^{\prime \prime} 3$ & 5.9 & $0.016( \pm 0.007)$ & $7.1( \pm 3.0) \times 10^{-13}$ & Unknown & $\mathrm{N}$ & 0 & 2 & 0 \\
\hline 8 & 4 & $22^{\mathrm{h}} 08^{\mathrm{m}} 54^{\mathrm{s}} .01+50^{\circ} 50^{\prime} 27^{\prime \prime} \cdot 4$ & 5.3 & $0.022( \pm 0.008)$ & $9( \pm 3) \times 10^{-13}$ & Star & Y & 1 & 1 & 0 \\
\hline 8 & 4 & $22^{\mathrm{h}} 15^{\mathrm{m}} 54^{\mathrm{s}} .39+52^{\circ} 18^{\prime} 37^{\prime \prime} 6$ & 6.1 & $0.015( \pm 0.006)$ & $6.5( \pm 2.8) \times 10^{-13}$ & Unknown & $\mathrm{Y}$ & 0 & 2 & 0 \\
\hline 8 & 4 & $22^{\mathrm{h}} 20^{\mathrm{m}} 06^{\mathrm{s}} .04+53^{\circ} 00^{\prime} 37^{\prime \prime} 6$ & 8.6 & $0.015( \pm 0.007)$ & $6.3( \pm 3.0) \times 10^{-13}$ & Unknown & $\mathrm{Y}$ & 1 & 6 & 0 \\
\hline 8 & 4 & $22^{\mathrm{h}} 20^{\mathrm{m}} 06^{\mathrm{s}} \cdot 76+49^{\circ} 30^{\prime} \quad 13^{\prime \prime} 3$ & 8.7 & $0.15( \pm 0.06)$ & $6.6( \pm 2.5) \times 10^{-12}$ & BYDra & $\mathrm{Y}$ & 0 & 1 & 0 \\
\hline 8 & 2 & $22^{\mathrm{h}} 21^{\mathrm{m}} 28^{\mathrm{s}} .16+50^{\circ} 32^{\prime} 44^{\prime \prime} \cdot 2$ & 6.2 & $0.015( \pm 0.007)$ & $6( \pm 3) \times 10^{-13}$ & Unknown & $\mathrm{N}$ & 0 & 1 & 4.4 \\
\hline 8 & 3 & $22^{\mathrm{h}} 28^{\mathrm{m}} 29^{\mathrm{s}} .95+53^{\circ} 44^{\prime} 10^{\prime \prime} .5$ & 5.4 & $0.034( \pm 0.010)$ & $1.4( \pm 0.4) \times 10^{-12}$ & Unknown & $\mathrm{N}$ & 0 & 1 & 5.2 \\
\hline 8 & 4 & $22^{\mathrm{h}} 29^{\mathrm{m}} 22^{\mathrm{s}} .74+53^{\circ} 49^{\prime} 44^{\prime \prime} 5$ & 5.3 & $0.032( \pm 0.010)$ & $1.4( \pm 0.4) \times 10^{-12}$ & Unknown & $\mathrm{Y}$ & 1 & 0 & 0 \\
\hline 8 & 3 & $22^{\mathrm{h}} 32^{\mathrm{m}} 11^{\mathrm{s}} .72+54^{\circ} 08^{\prime} \quad 11^{\prime \prime} 2$ & 4.7 & $0.021( \pm 0.008)$ & $9( \pm 3) \times 10^{-13}$ & Unknown & $\mathrm{N}$ & 1 & 2 & 0 \\
\hline 8 & 4 & $22^{\mathrm{h}} 32^{\mathrm{m}} 37^{\mathrm{s}} .82+54^{\circ} 05^{\prime} 29^{\prime \prime} 5$ & 5.6 & $0.067( \pm 0.014)$ & $2.9( \pm 0.6) \times 10^{-12}$ & Star & Y & 1 & 1 & 0 \\
\hline 8 & 4 & $22^{\mathrm{h}} 35^{\mathrm{m}} 40^{\mathrm{s}} .00+53^{\circ} 45^{\prime} 31^{\prime \prime} 3$ & 6.2 & $0.05( \pm 0.04)$ & $2.3( \pm 1.8) \times 10^{-12}$ & AGN candidate & $\mathrm{Y}$ & 1 & 1 & 1.2 \\
\hline 4 & 4 & $23^{\mathrm{h}} 21^{\mathrm{m}} 15^{\mathrm{s}} .75-26^{\circ} 58^{\prime} 55^{\prime \prime} .8$ & 7.6 & $0.26( \pm 0.06)$ & $1.12( \pm 0.26) \times 10^{-11}$ & Unknown & $\mathrm{Y}$ & 1 & 0 & 0 \\
\hline
\end{tabular}

Note. The following columns are given: GW \#-the number of the GW event (4 corresponds to the first entry in Table 1 , and 17 corresponds to the last); Rank-as described in Section 3 ( 2 = interesting source, $3=$ uncataloged X-ray source, $4=$ cataloged X-ray source); R.A./decl. (J2000); Err-90\% positional uncertainty; Peak Rate—peak source XRT count rate; Peak Flux (see Section 3 for details); SIMBAD Src Type—(see http://simbad.u-strasbg.fr/Pages/guide/chF.htx for details; "AGN; MQ" and "AGN; S/W" denote that there is a positionally coincident entry in the Million Quasars catalog or the QSO selection from the Sloan Digital Sky Survey and WISE; see Flesch 2015 and Richards et al. 2015, respectively); Known X-whether or not the source has been previously detected in X-rays; Near Gal.the number of known "nearby" galaxies (see Section 4.1 for details); Near 2MASS - the number of nearby/positionally coincident 2MASS sources; Fading - the statistical significance of fading behavior, if present.

(This table is available in machine-readable form.)

\section{A.4. G275404/LVC 170225}

LVC detected a CBC trigger at 18:30:21 UT, with an estimated distance $d=412 \pm 169 \mathrm{Mpc}$. It was reported as having a probability of being EM-bright of 0.9 and a $P_{\mathrm{NS}}=1$. The first PPST list was uploaded at 23:08 UT, and the first Swift observation occurred at T0+17.3 ks. Of the 1402 planned tiles, 117 were observed, covering $1.8 \%$ of the raw (and $4 \%$ of the convolved) LVC probability region. Three X-ray sources were detected: one known (rank 4) source and two unlikely afterglows (rank 3 sources). The trigger was revised on 02/ 26-00:20 and again at 02/26-06:16. Phase 1 was carried out, but trigger G275697 occurred when phase 2 was due to start, so we decided to prioritize the more recent and nearby event (described in following section).

Three GCN Circulars were sent by the Swift team. GCN 20746: the BAT covered $18.68 \%$ of the LVC probability region at the time of trigger, but no significant detections were found.
GCN 20752: a report on the above-mentioned XRT sources. GCN 20842: a report on the XRT follow-up of the possible counterparts coincident with the position of the transient reported by AGILE, AGL J1914+1043: GL 191032+075314 and GRS 1915+105.

\section{A.5. G275697/LVC 170227}

LVC detected a CBC trigger at 18:57:31 UT, with an estimated distance $d=193 \pm 61 \mathrm{Mpc}$. It was reported as having a probability of being EM-bright of 1 . Of the 1414 planned tiles, 1408 tiles were observed. This is the first trigger for which phase 1 was carried out in full (e.g., without a reduction to the number of tiles), and the first (and only) trigger for which the full three-phase observing plan was carried out. No negative effects on the spacecraft (e.g., due to the high rate of slewing) were recorded. The trigger was subsequently retracted, after our follow-up search was performed. 
Eight GCN Circulars were sent by the Swift team. GCN 20772: the BAT covered $8 \%$ of the LVC probability region at the time of trigger, but no significant detections were found. GCNs 20773, 20798, 20807, 20812, and 20821 were reports on sources found by the XRT. GCNs 20841 and 20884: the XRT team reported on the observed fading and subsequent cessation of fading of a source.

\section{A.6. G277583/LVC 170313}

LVC detected an unmodeled burst trigger at 22:40:09 UT. No tiling was performed, since the trigger did not meet our trigger criteria. The BAT covered $19.2 \%$ of the LVC probability region at trigger time, but no significant detections were found (GCN 20883).

\section{A.7. G284239/LVC 170502}

LVC detected an unmodeled burst trigger at 22:26:07 UT. No tiling was performed, since the trigger did not meet our trigger criteria. $0 \%$ of the LVC probability region was covered by the BAT at the time of trigger, and no significant detections were found (GCN 21065).

\section{A.8. G288732/LVC 170608}

LVC detected a CBC trigger at 02:01:16 UT, with an estimated distance $d=320 \pm 98 \mathrm{Mpc}$. No tiling was initially performed, since the trigger did not meet our trigger criteria. However, a 4-point tiling was performed at $\mathrm{T} 0+62.8 \mathrm{ks}$ to follow up a possible counterpart detected by the Fermi-LAT. Seven X-ray sources were detected: two known (rank 4) sources and five unlikely afterglows (rank 3 sources).

Three GCN Circulars were sent by the Swift team. GCN 21233: described the XRT sources detected in the follow-up of the LAT source. GCN 21234: no significant detections were found in the BAT data (the BAT covered $0 \%$ of the LVC probability region at the time of trigger). GCN 21235: summarized the UVOT follow-up; no compelling sources were found.

The following day the region of sky entered Swift's Sun observing constraint (until September), so no further follow-up was conducted.

\section{A.9. G296853/LVC 170809}

LVC detected a CBC trigger at 08:28:21 UT, with an estimated distance $d=1086 \pm 302 \mathrm{Mpc}$. No tiling was performed, since the trigger did not meet our trigger criteria. $0 \%$ of the LVC probability region was covered by the BAT at the time of trigger (GCN 21436).

\section{A.10. G297595/LVC 170814}

LVC detected a CBC trigger at 10:30:43 UT, with an estimated distance $d=534 \pm 131 \mathrm{Mpc}$. The first PPST list was uploaded at 18:22 UT, and the first observation occurred at $\mathrm{T} 0+31.2 \mathrm{ks}$. Of the 726 planned tiles, 643 were observed, covering $24 \%$ of the raw (and $36 \%$ of the convolved) LVC probability region. A total of $41 \mathrm{X}$-ray sources were detected: 26 known (rank 4) sources and 15 unlikely afterglows (rank 3 sources).

Two GCNs were sent by the Swift team. GCN 21483: 0\% of the LVC probability region was covered by the BAT at the time of trigger; a $5.4 \sigma$ spike was detected at $\mathrm{T} 0+27 \mathrm{~s}$, but it is likely noise. GCN 21503: reported on the XRT sources.

\section{A.11. G298048/LVC 170817}

LVC detected a CBC trigger at 12:41:04 UT, with an estimated distance $d=39 \pm 7 \mathrm{Mpc}$. The first PPST list was promptly uploaded, and the first observation occurred at T0 $+3.3 \mathrm{ks}$. Of the 2966 tilings planned, 744 were observed, covering $2.5 \%$ of the raw (and $94 \%$ of the convolved) LVC probability region. This was the epochal GW170817, the first GW event for which the EM counterpart was discovered and identified. Swift and NUSTAR observations of this event are discussed in depth by Evans et al. (2017).

\section{A.12. G298389/LVC 170819}

LVC detected an unmodeled burst trigger at 15:50:46 UT. No tiling was performed, as we decided to prioritize the followup search for the previous trigger (GW170817). $0 \%$ of the LVC probability region was covered by the BAT at the time of trigger, and no significant detections were found (GCN 21622).

\section{A.13. G298936/LVC 170823}

LVC detected a CBC trigger at 13:13:58 UT, with an estimated distance $d=1738 \pm 477 \mathrm{Mpc}$. No tiling was performed, as the source was too far away for galaxy targeting, the event was a $\mathrm{BBH}$, and we were still following up with GW170817. $9.5 \%$ of the LVC probability region was covered by the BAT at the time of trigger; a $5.1 \sigma$ spike was detected at $\mathrm{T} 0-30 \mathrm{~s}$, but it was probably not astrophysical in origin (GCN 21665).

\section{A.14. G299232/LVC 170825}

LVC detected a CBC trigger at 13:13:37 UT, with an estimated distance $d=339 \pm 109 \mathrm{Mpc}$. The first PPST list was uploaded at 15:32 UT, and the first Swift observation was carried out at $\mathrm{T} 0+11.1 \mathrm{ks}$. Of the 1096 tilings planned, 653 were observed, covering $8.3 \%$ of the raw (and $16.0 \%$ of the convolved) LVC probability region. The list of tiles for this plan had to be reduced owing to XRT temperature considerations. A total of $51 \mathrm{X}$-ray sources were detected: 1 interesting (rank 2) source, 30 unlikely afterglows (rank 3 sources), and 20 known (rank 4) sources. The rank 2 source was 1RXS J014709.9+234529 (an RS CVn variable star).

Four GCN Circulars were sent by the Swift team. GCN 21704: $11.56 \%$ of the LVC probability region was covered by BAT at the time of trigger, but no significant detections were found. GCN 21733: the optical transient Swift J014008.5 +343403.6 was discovered with the UVOT at magnitude 18 , but with no X-ray counterpart. GCN 21758: the UVOT counterpart was observed again but was not detected. GCN 21844: reported on the XRT sources.

\section{ORCID iDs}

N. J. Klingler (iD https://orcid.org/0000-0002-7465-0941

J. A. Kennea (iD https://orcid.org/0000-0002-6745-4790

P. A. Evans (i) https://orcid.org/0000-0002-8465-3353

A. Tohuvavohu (10 https://orcid.org/0000-0002-2810-8764

S. B. Cenko (D) https://orcid.org/0000-0003-1673-970X

A. A. Breeveld (1D https://orcid.org/0000-0002-0001-7270 
P. J. Brown (1) https://orcid.org/0000-0001-6272-5507

D. N. Burrows (1) https://orcid.org/0000-0003-0729-1632

A. D'Aì (i) https://orcid.org/0000-0002-5042-1036

J. Garcia (16) https://orcid.org/0000-0003-3828-2448

D. H. Hartmann (1) https://orcid.org/0000-0002-8028-0991

D. B. Malesani (i) https://orcid.org/0000-0002-7517-326X

F. E. Marshall ๑i https://orcid.org/0000-0002-3559-6305

J. P. Osborne (1) https://orcid.org/0000-0002-1041-7542

K. L. Page (i) https://orcid.org/0000-0001-5624-2613

D. M. Palmer (1) https://orcid.org/0000-0001-7128-0802

J. L. Racusin (1) https://orcid.org/0000-0002-4744-9898

G. Tagliaferri (i) https://orcid.org/0000-0003-0121-0723

E. Troja (1) https://orcid.org/0000-0002-1869-7817

\section{References}

Abbott, B. P., Abbott, R., Abbott, T. D., et al. 2017, ApJL, 848, L12 Abbott, B. P., Abbott, R., Abbott, T. D., et al. 2019a, ApJ, 875, 161 Abbott, B. P., Abbott, R., Abbott, T. D., et al. 2019b, PhRvX, 9, 031040

Acernese, F., Agathos, M., Agatsuma, K., et al. 2015, CQGra, 32, 024001

Barthelmy, S. D., Barbier, L. M., Cummings, J. R., et al. 2005, SSRv, 120, 143

Beniamini, P., Petropoulou, M., Barniol Duran, R., \& Giannios, D. 2019, MNRAS, 483, 840

Beniamini, P., \& van der Horst, A. J. 2017, MNRAS, 472, 3161

Berger, E. 2014, ARA\&A, 52, 43

Berger, E., Kulkarni, S. R., \& Frail, D. A. 2003, ApJ, 590, 379

Bilicki, M., Jarrett, T. H., Peacock, J. A., Cluver, M. E., \& Steward, L. 2014, ApJS, 210, 9

Bloom, J. S., Frail, D. A., \& Sari, R. 2001, AJ, 121, 2879

Bulik, T., Belczyński, K., \& Zbijewski, W. 1999, MNRAS, 309, 629

Burrows, D. N., Hill, J. E., Nousek, J. A., et al. 2005, SSRv, 120, 165

D'Avanzo, P. 2015, JHEAp, 7, 73

Eichler, D., Livio, M., Piran, T., \& Schramm, D. N. 1989, Natur, 340, 126

Evans, P. A., Beardmore, A. P., Page, K. L., et al. 2009, MNRAS, 397, 1177

Evans, P. A., Cenko, S. B., Kennea, J. A., et al. 2017, Sci, 358, 1565

Evans, P. A., Kennea, J. A., Barthelmy, S. D., et al. 2016a, MNRAS, 460, L40

Evans, P. A., Kennea, J. A., Palmer, D. M., et al. 2016b, MNRAS, 462, 1591 Evans, P. A., Kennea, J. A., Palmer, D. M., et al. 2019, MNRAS, 484, 2362

Evans, P. A., Osborne, J. P., Beardmore, A. P., et al. 2014, ApJS, 210, 8
Evans, P. A., Osborne, J. P., Kennea, J. A., et al. 2015, MNRAS, 448, 2210 Evans, P. A., Osborne, J. P., Kennea, J. A., et al. 2016c, MNRAS, 455, 1522 Flesch, E. W. 2015, PASA, 32, e010

Fong, W., Berger, E., Chornock, R., et al. 2013, ApJ, 769, 56

Fong, W., Berger, E., \& Fox, D. B. 2010, ApJ, 708, 9

Fong, W., Berger, E., Margutti, R., \& Zauderer, B. A. 2015, ApJ, 815, 102

Frail, D. A., Kulkarni, S. R., Sari, R., et al. 2001, ApJL, 562, L55

Gehrels, N., Chincarini, G., Giommi, P., et al. 2004, ApJ, 611, 1005

Ghirlanda, G., Salafia, O. S., Paragi, Z., et al. 2019, Sci, 363, 968

Haggard, D., Nynka, M., Ruan, J. J., et al. 2017, ApJL, 848, L25

HEASARC 2014, HEASoft, Astrophysics Source Code Library, ascl:1408.004

Kamble, A., \& Kaplan, D. L. A. 2013, IJMPD, 22, 1341011

Kanner, J., Baker, J., Blackburn, L., et al. 2013, ApJ, 774, 63

Li, L.-X., \& Paczyński, B. 1998, ApJL, 507, L59

LIGO Scientific Collaboration, Aasi, J., Abbott, B. P., et al. 2015, CQGra, 32 , 074001

LIGO Scientific Collaboration, \& Virgo Collaboration 2019, arXiv:1907.01443

Liu, T., Romero, G. E., Liu, M.-L., \& Li, A. 2016, ApJ, 826, 82

Loeb, A. 2016, ApJL, 819, L21

Margutti, R., Alexander, K. D., Xie, X., et al. 2018, ApJL, 856, L18

Margutti, R., Berger, E., Fong, W., et al. 2017, ApJL, 848, L20

Metzger, B. D., \& Berger, E. 2012, ApJ, 746, 48

Metzger, B. D., Martínez-Pinedo, G., Darbha, S., et al. 2010, MNRAS, 406,2650

Middei, R., Vagnetti, F., Bianchi, S., et al. 2017, A\&A, 599, A82

Perna, R., Lazzati, D., \& Giacomazzo, B. 2016, ApJL, 821, L18

Richards, G. T., Myers, A. D., Peters, C. M., et al. 2015, ApJS, 219, 39

Roming, P. W. A., Kennedy, T. E., Mason, K. O., et al. 2005, SSRv, 120, 95

Ryan, G., van Eerten, H., Piro, L., \& Troja, E. 2019, arXiv:1909.11691

Saxton, R. D., Read, A. M., Esquej, P., et al. 2008, A\&A, 480, 611

Schutz, B. F. 2011, CQGra, 28, 125023

Sisteró, R. F., \& Castore de Sisteró, M. E. 1974, AJ, 79, 391

Sun, H., Zhang, B., \& Gao, H. 2017, ApJ, 835, 7

Troja, E., Piro, L., Ryan, G., et al. 2018, MNRAS, 478, L18

Troja, E., Piro, L., van Eerten, H., et al. 2017, Natur, 551, 71

Tunnicliffe, R. L., Levan, A. J., Tanvir, N. R., et al. 2014, MNRAS, 437, 1495

van Eerten, H. 2018, IJMPD, 27, 1842002-314

van Eerten, H., Zhang, W., \& MacFadyen, A. 2010, ApJ, 722, 235

Voges, W., Aschenbach, B., Boller, T., et al. 1999, A\&A, 349, 389

White, D. J., Daw, E. J., \& Dhillon, V. S. 2011, CQGra, 28, 085016

Yamazaki, R., Asano, K., \& Ohira, Y. 2016, PTEP, 2016, $051 \mathrm{E} 01$

Zhang, B. 2016, ApJL, 827, L31 